\title{
Non Invasive Acoustic Measurements for Faults Detecting in Building Materials and Structures
}

\author{
Barbara De Nicolo, Carlo Piga, Vlad Popescu and Giovanna Concu \\ University of Cagliari, Engineering Faculty, \\ Italy
}

\section{Introduction}

\subsection{Backgrounds and generalities}

Over the past years both large and small restoration and conservation works on monuments, civil and industrial buildings have become of great interest. As indicators of the historical period in which they were built, all construction works have both their architectural style and the material used in their construction. Indeed, for thousands of years humans built using the same materials (wood, stone, brick, mortar and gypsum) up to the introduction of concrete at the beginning of the $19^{\text {th }}$ century. Although concrete has replaced the old materials used in historical buildings, there still remains the problem of forestalling their deterioration and restoring them, also in the light of the importance of such works from the historical, cultural and economic viewpoints. Problems connected with the restoration of buildings, whether in reinforced concrete, masonry or wood, are quite complex and are essentially linked to the reuse, and thus the redesign, of the existing heritage of buildings. Indeed, cultural, social and economic reasons foster to the desire to lengthen the life of this heritage beyond normal physiological limits and thus its fruition far beyond its useful life. The problems to be addressed vary widely since there are noteworthy differences from one job to another; it is sufficient to consider just the social value of a building of great historical value, which is usually protected by severe restrictions aimed at conserving its artistic and cultural features, or an industrial building the use of which must be completely changed while at the same time maintaining its structural characteristics. It is evident that there is not one single answer to such widely differing situations: each job must be addressed from the cultural, technological and technical standpoints as a special case. The proper management of the rehabilitation of a building implies a knowledge of its real static conditions to be restored, the mechanical, physical and chemical characteristics of the materials of which it is built and the presence and characteristics of defects, anomalies and so on. Of fundamental importance is the diagnosis of materials and structures and many researchers, as well as companies that produce restoration materials, have carried out studies in this field. Methods for structural diagnosis and faults detecting are beginning to appear, albeit in an extremely divergent way, in tenders, rules, guidelines and so on. To exemplify, non-invasive diagnostic techniques are often used to determine whether or not materials compatible with the original structure have been used in restoration works: if not, 
the building may undergo greater damage than that caused by progressive deterioration. All the factors illustrated above favour the development of new control and diagnostic techniques that produce more and more information on the state of the structure and, indirectly, represent a more precise instrument for use in the planning of restoration works.

In the field of structural faults detection particular importance is given to developments of Non-Destructive Testing measurements techniques (NDT), including automated procedures and information technology to support decision making and evaluation of data. NDT appear to be of great usefulness since, compared to classic laboratory techniques, they are non-invasive, faster and of a general rather than specific nature. These skills have led to the creation, evolution and rapid diffusion of certain diagnostic non-destructive measurements techniques. The main obstacle to the effective, systematic and economical use of NDT in structural diagnostics lies in the gap that exists between the theoretical and interpretative bases and codification of operative modalities, which is to say the almost total lack of standardization procedures; moreover, practical experience has underscored the limits of commercial devices, which have often proved to be incapable of adapting to specific structural problems under investigation and have interfaces that do not allow users to check the validity of data acquired and the accuracy of measurements, thus making the results aleatory and difficult to interpret.

As a major NDT tool, acoustic techniques, based on measurements of the characteristics of acoustic waves propagating through the material, are often used in quality control and faults detection for engineering structures and infrastructures. The studies of acoustic techniques have been focused on medical or materials engineering applications for laboratory testing. The valuable handmade analysis has begun in the first of 190 and nowadays it is performed using considerable approximations to the detriment of results precision. The inaccurate results are also caused by the subjective methodology often used for interpretations. This contributed to form the common opinion that the use of such methodologies in structural diagnostics does not give reliable results. Conversely, the aforesaid techniques could show notable diagnostic properties if used in an appropriate way, that is providing them with innovative techniques, which employ and develop advanced computational tools and testing devices. In the light of this, fuelled by the rapid development of portable personal computers, high-performance computing algorithms and electronic engineering technology, acoustic techniques have evolved dramatically during recent years.

Acoustic material analysis is based on a simple principle of physics: the propagation of any wave will be affected by the medium through which it travels. Thus, changes in measurable parameters associated with the passage of a wave through a material can be correlated with changes in physical properties of the material. Recently, thanks to continuing scientific and technological progress, systematic studies and applications of such methods in many different fields have been developed. The application of methods of automated graphic representation to the so-called media transparent to vibrations such as even large metal pieces, has now become routine. Outside the field of engineering, such methods have also been applied to parts of the human body; it is in the techniques of medical analysis such as ultrasonography and computerized axial tomography that some of the most important methods of investigation of structures with acoustics NDT have been developed. The 
acoustic NDT of materials less transparent to vibrations, such as concrete, mortars, stones, wood, masonry and so on, is still more challenging. For such substantially inhomogeneous media, the development of acoustic testing techniques has been decidedly slower compared to applications on transparent media. This depends mainly on the greater difficulties and the important theoretical and interpretative challenges, not to mention the technological ones represented by the reduced transparency of the materials to vibrations deriving from their high degree of intrinsic lack of homogeneity.

\subsection{Methodologies}

\subsubsection{Sonic and ultrasonic testing methods}

Sonic and ultrasonic investigations refer to a complex method for the analysis of materials and the structures of which they are made, based on the study of phenomena connected with the propagation of elastic perturbations inside the materials under study. The signal that penetrates into the material is generated artificially by an external source and acquired by means of a receiver after passing through the medium following appropriate trajectories. From analysis of the processes and parameters connected with the propagation of acoustic perturbations inside the artefact it is possible to collect a great deal of information on the material or structure under study (J. Krautkramer \& H. Krautkramer, 1990). This information includes:

- the level of homogeneity of the material concerning several elements or a certain number of samples or only one of these;

- the quality and degree of deterioration of the material;

- the estimate of certain elastomechanical characteristics;

- the identification of possible faults in the material or structure, such as cavities, inclusions and zones having different elastomechanical characteristics;

- the trend in time of different phenomena and related to the stresses the materials undergo.

The intrinsic characteristics of the medium under test intervene decidedly in the several aspects connected with the use of acoustic methods (choice of the most suitable instrumentation, application of the basic principles of the method, criteria for interpreting the results), especially in the choice of the signal frequency to use in the investigation.

In the case of not large homogeneous media, for example, metal pieces, the characteristics of homogeneity favour the use of ultrasonic frequencies above $500 \mathrm{KHz}$. The absence of natural non homogeneities allows the signal to propagate without appreciable reflection, refraction or mode conversion diffusion phenomena, unless these are caused by the presence of possible local anomalies. The analysis of such kinds of media is thus favoured by the negligible attenuation of the signal. This requires the use of very little energy and makes it possible to identify even very small defects by emitting very narrow irradiation bands through strongly directional probes. Substantially, in these cases it is possible to plan the analysis in the smallest details and accurately investigate by closely defined zones from the geometric standpoint.

For investigations in media with a high degree of non homogeneity, for example the stratigraphic analysis of terrains, due to the strong intrinsic heterogeneity of the medium 
there is a noteworthy diffusion of the signal. This, together with the need to investigate large areas, calls for the use of sources with high emission energy and therefore kept prevalent in low frequencies (usually $5-150 \mathrm{~Hz}$ ), so as to compensate for the high absorption characteristics of the medium and the total loss of signal direction.

When intrinsically inhomogeneous media of not very large dimensions such as those represented by building structures are to be investigated, the problem of revealing perturbations caused by anomalies of great interest arises; however, such anomalies differ slightly in extension, geometry and dimensions from the natural non homogeneities of the medium under study: an emblematic case is that of concrete in which the anomalies may respond to the acoustic input in a way similar to the aggregate. For this category of media, in which we find most of the materials and structures used in civil, industrial and monumental engineering and architecture, it is of fundamental importance to choose correctly the characteristics of the signal employed, attempting a mediation between the characteristics of the ultrasonic signals (enhanced diagnostic precision at the price of greater attenuation and thus a lesser penetrative capacity) and that of the sonic signals (high penetrative capacity at the price of poor definition). This is the case of concrete elements, masonry structures, wood elements and limited volumes of terrain.

Experimental results (Concu et al., 2003a) showed that both sonic and ultrasonic signals reveal the presence of macroscopic anomalies inside a limestone masonry structure, but are differently sensitive to intrinsic structural characteristics and environmental conditions. We performed the experiment on a sample masonry wall of limestone blocks, inside which some metal and wood elements, assumed as anomalies of the structure, were placed in a known position. Moreover, in a central position of the masonry a cavity was excavated designed to hold a rectangular section, but the irregularity of the external blocks and the intrusion of mortar caused the section to become irregular in the executive stage. The propagation velocity of high- and low-frequency signals through the wall thickness was measured: higher velocities are generally associated with a better quality of the material. Velocity data were then elaborated by interpolation and represented in the form of a map of velocity distribution in a generic vertical section of the wall. Fig. 1 shows the velocity maps obtained with the sonic (low-frequency signals) and ultrasonic (high-frequency signals) methods.
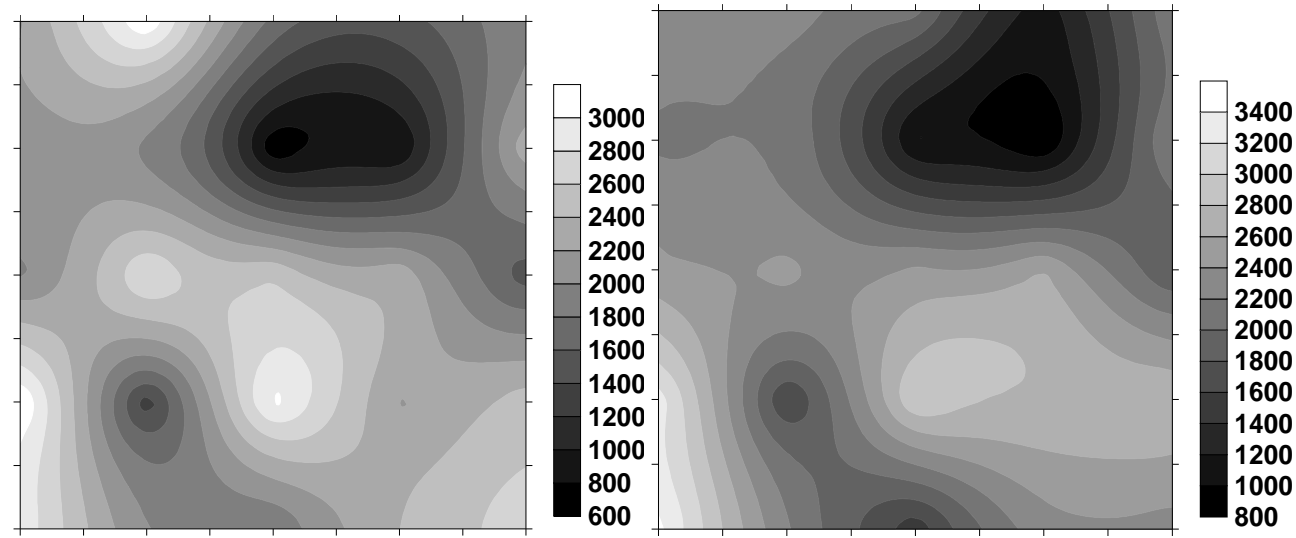

Fig. 1. Maps of velocity [m/s]. Sonic velocity (left) and ultrasonic velocity (right). Extracted from Concu et al., 2003a 
The two velocity distributions appear quite similar. Substantially, the two methods show the same velocity range, with maxima and minima located in the same areas. Both maps reveal a well-defined region of minimum velocity in correspondence to the cavity. These results underscore that both methods are useful for revealing the presence of macroscopic anomalies inside stone masonry. The test also made it possible to demonstrate that environmental noises and vibrations impact more on sonic than on ultrasonic method; the latter is instead less practical since it calls for the use of a transducer rather than a hammer as the source, and this requires the precise positioning of source and receiver on the surface of the structure before each signal acquisition.

Independently of the frequency characteristics of the signal employed in the investigation, the parameters associated with the signal penetrating through the medium are the following:

- transit time (or travel time), that is, the time taken by the signal to cover the distance from the source to the receiver inside the material under examination;

- signal propagation velocity, in the sense of the ratio of the distance between source and receiver to transit time;

- $\quad$ signal attenuation characteristics in its passage through the material.

Traditional application of acoustic techniques is based on measurements of the velocity $\mathrm{V}$ of acoustic waves propagating through the material. The velocity is obtained from the ratio $\mathrm{L} / \mathrm{T}$, where $\mathrm{T}$ is the time wave needs to travel along the path of length $\mathrm{L}$. The wave velocity is directly related to structure's elastic parameters, e.g. elastic modulus E, Poisson's number $v$ and density $\rho$, thus its analysis provides information crucial for inspections of structures inner conditions.

The propagation velocity, although a significant parameter, limits the investigation at the analysis of propagation times, not taking into consideration important information regarding the way the waves are propagating. For instance, when a wave passing through a specific item encounters any discontinuity, the wave power is certainly attenuated because of scattering phenomena, while the propagation time may not be moved if part of the signal is already able to reach the receiver. Therefore, it would be reasonable to approach the acoustic analysis also in terms of other wave's features changes and not only in terms of propagation times. The higher the intrinsic non-homogeneity level of structures, e.g. masonries, the bigger the advisability of this integrated approach. In fact, as documented by various studies predominantly performed in the geophysics and aeronautics environments, other wave's characteristics such as attenuation, scattering and frequency content, primarily related to the elastic wave power, may allow one to get more and relevant information about the material, because of the reliance of the propagation on the properties of the medium through which waves travel. In fact, different materials absorb or attenuate the wave power at different rates, depending on complex interactive effects of material characteristics, such as density, viscosity, homogeneity. Additionally, waves are reflected by boundaries between dissimilar materials, so that changes in materials structure, e.g. presence of discontinuities or defects, can affect amplitude, direction, and frequency content of scattered signals. Furthermore, all materials behave somehow as low pass filters, attenuating or scattering the higher frequency components of a broadband wave more than the lower. Thus, waves analysis in terms of multiple wave's features can give information on the combined effects of attenuation and scattering as previously described. 
The comparative analysis of transit time and amplitude attenuation of acoustic signals carried out on the stone wall previously described confirmed the opportuneness of the joint use of both parameters (Concu et al., 2003b). In this study, we assumed the amplitude attenuation as the ratio of received signal to transmitted signal maximum amplitude. Fig. 2 shows the map of transit time and amplitude attenuation in the generic vertical section of the masonry.
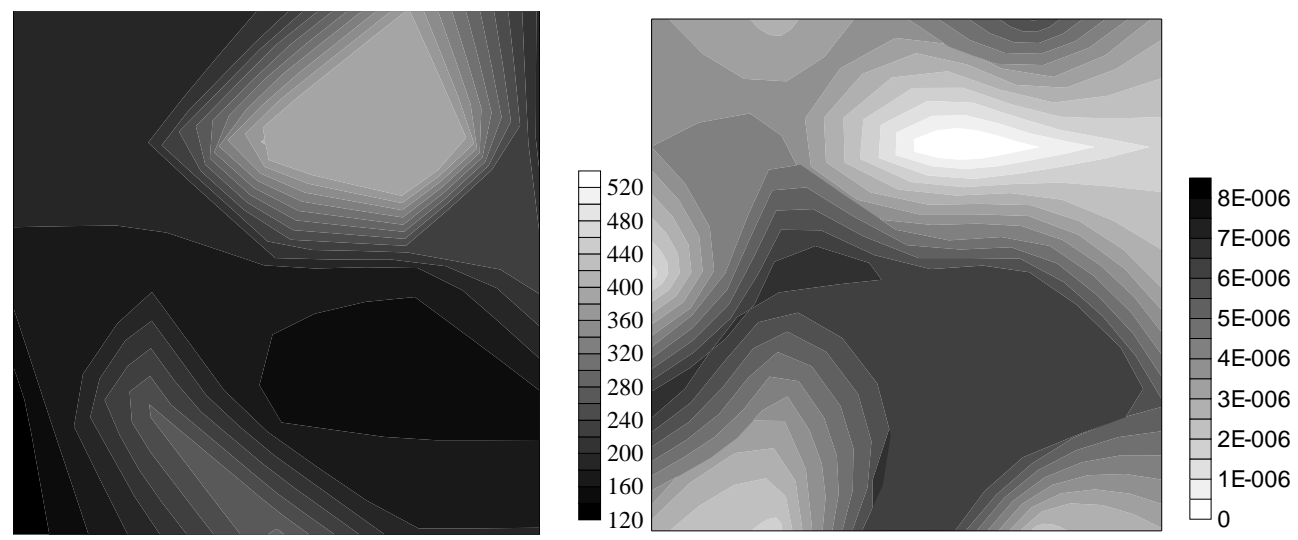

Fig. 2. Map of transit time [ $\mu \mathrm{s}]$ (left) and amplitude attenuation (right). Extracted from Concu et al.,2003b

By observing the map we can see that the distribution of transit time shows a clearly defined section with maximum values in association with the cavity inside the wall. This section can be illustrated as a geometric figure of size and position perfectly compatible with the design geometry of the cavity before erection of the wall. Distribution of times in the rest of the map appears on the whole uniform. From this we can deduce that ultrasonic signal transit time (thus velocity) is a parameter capable of identifying macroscopic defects with a good degree of approximation and immediacy, while it does not appear to be sensitive to minor anomalies such as the absence of material between the stone blocks, the presence of mortar joints or small elements of different material. Interpretation of distribution concerning amplitude attenuation is less immediate: we can see a zone of maximum attenuation in correspondence to the cavity, the borders of which are rather blurred. Attenuation values are on the whole rather dispersed. This confirms that amplitude attenuation is a parameter extremely sensitive to all kinds of discontinuities of the material that cause a loss of signal energy and it is therefore quite suitable for use in tests at high definition in small areas. Transit time and amplitude attenuation thus have different diagnostic capabilities and this emphasizes the usefulness of different wave's features integrated use in structural faults diagnoses.

The study of energy characteristics associated with the signal is today addressed also by employing the spectral analysis. By spectral analysis is meant the method based on analysis of the frequency content of the signal travelling through the material of interest. When a signal goes through a medium, the frequency components associated with the input signal are altered since the medium acts as a filter that transmits only a certain frequency band with different degrees of attenuation and phase shifts. It is thus possible to study the effects of the medium's properties on alteration of the input signal by performing a spectral 
analysis on the signals passing through different materials or different portions of the same material or the same material in different surrounding conditions: a given input signal will emerge with different spectrum frequencies after passing through materials with different characteristics (Priestley, 1981). Substantially, the spectral analysis provides a sort of signature characteristic of the properties of the material travelled through. The material is gone through by a signal of the impulsive kind expressed by means of a function of type $\mathrm{A}(\mathrm{t})$, in which the oscillation amplitudes are given as a function of time. The spectrum of the signal offers instead a representation of the same in the frequency domain, in which the signal is expressed by means of a function of type a(f): the amplitudes of the elementary oscillations that make up the impulse are given as a function of the respective frequencies.

\subsubsection{Acoustic emission}

The acoustic emission technique is based fundamentally on the study of the acoustic signal emitted by the material during deformation, cracking, breaking, collapse and in general during whatever phase causing a release of energy. The single event, that is, the single emission, is thus an impulsive acoustic signal produced by a source within the material following the triggering of any phenomenon capable of releasing energy. The main parameters associated with acoustic emissions and used for the study and application of the method in the field of structural monitoring and faults detection are:

- number of events emitted during the phenomenon - deformation, loading and so on under observation;

- emission velocity, in the sense of the number of events in a given time interval;

- maximum amplitude of the event;

- duration of the event;

- wave shape of the event;

- frequency spectrum of the event;

- $\quad$ arrival time of the signal at the transducers used for establishing it;

- the so-called "b-value", that is, the slope of the logarithmic curve representing the maximum amplitude of the events as a function of the frequency with which they appear.

These parameters are placed together with the factors that identify the specific issue being tested, such as the stress-deformation load diagrams, microscopic observations and so on, so as to find a relation that makes it possible to arrive at the state of the material starting from observation of the magnitudes associated with its acoustic emission.

The potentialities of the method for the study and monitoring of the behavior of materials, the prediction of their response to different kinds of stresses and the check of defects and anomalies are many. They are based on special phenomena, such as the Kaiser effect, on the application of instruments of mathematical analysis and numerical calculus, and on the comparative study of acoustic parameters and elastomechanical characteristics (Enoki \& Kishi, 1991).

The Kaiser effect (Kaiser, 1950) is a phenomenon by which a material under stress emits acoustic signals which are significant only when the level of stress to which the material was previously submitted has been exceeded. In effect, there are emissions even below this level, but the two kinds of events differ greatly: in rapid succession and high energy content the 
former, associated with the triggering and propagation of new cracks; less frequent and with much lesser amplitudes the latter, associated with the deformation and contact between the surfaces of cracks opened in the previous load cycle. Substantially, the material has a recollection of its history of load and deformation. The uses of this phenomenon are many. It is used to arrive at the maximum stress that a material has undergone, for example the original stress of a rock, estimated by submitting to loading and unloading test samples taken from the rock mass. It is exploited in identifying the breakage surface associated with the material in different load conditions. It can be an indicator of the state of deterioration of the material, for example, of the damage caused to it by loads, since emissions of the second type appear for lower load levels in the most damaged materials.

The possibility of characterizing the source of acoustic emissions arises on using a suitable number of transducers (in any case more than three): it is in fact possible to return to the position of the signal source by recording its arrival times at the different transducers and knowing the value of the characteristic acoustic velocity of the material. By interpolation we find the point inside the material which, for the assigned velocity, satisfies the values of all the times recorded by the transducers. By means of a well-documented mathematical treatment based on Green's functions, on the analysis of tensorial moments and on the calculation of eigenvalue and eigenvector it is possible to identify not only the position but also the volume and spatial orientation of the source inside the medium, thus obtaining its complete geometric characterization (Ohtsu \& Ono, 1986).

The comparative study of the acoustic emission parameters and factors of the elastomechanical type makes it possible to obtain many supplementary data on the behavior of the material. Acquisition of the acoustic emission parameters associated with knowledge of the diagrams of load, stress and deformation, as well as the characteristics of the material observed under the microscope before, during and after the phenomena under examination leads to the definition of precise correspondences between acoustic parameter values and certain fundamental data such as:

- level of load, stress and deformation;

- $\quad$ type of defect originating the emission (intra-, inter- and transgranular cracks);

- way of propagation of breaks (tensile or shear) and thus the kind of breakage observed;

- level of creep and the way in which it develops;

- dimension of the damaged zone in the material and so on.

From this naturally derives also the possibility of foreseeing the responses of the material to different kinds of stress, starting from the analysis of its acoustic emission. Substantially, the study of acoustic emissions has great potentialities in the monitoring of complex civil, industrial or natural structures, of single structural elements or laboratory test pieces made up of many different materials such as metals, cement, rock, ceramics, synthetic materials and so on. Interest concerning the use of the acoustic emission technique is proven also by the presence on the market of many kinds of often quite complex instrumental sets capable of acquiring and processing acoustic emission data in real time and proposing diagnostic hypotheses on the materials or structure under investigation by means of comparisons with databases on acoustic parameters. The application of this methodology is of great service in real-time monitoring of the triggering and propagation of breaks in materials in use as well as in the characterization of their behavior and the prediction of their responses to various stresses. 


\section{Operative procedures}

\subsection{Direct and indirect transmission}

The easier and faster way to get relevant information using sonic and ultrasonic methods is the measurement of the waves propagation velocity V. In fact, the operative procedure to acquire waves velocity and to process data in order to get immediate results is quite simple. In addition, the skills of this parameter are very useful: in fact, from wave's propagation theory it is known that $\mathrm{V}$ is dependent on the following material's characteristics: dynamic elastic modulus $E_{d}$, Poisson's number $v$ and density $\rho$. For a homogeneous isotropic material this function is:

$$
\mathrm{V}=\left[\frac{\mathrm{E}_{\mathrm{d}}}{\rho} \frac{(1-\mathrm{v})}{(1+\mathrm{v})}(1-2 \mathrm{v})\right] \frac{1}{2}
$$

$\mathrm{V}$ is directly related to structures elastic parameters, so that it has been frequently applied for evaluating structures integrity and restoration's effectiveness. Thus the relation between $V$ and $E_{d}, v, \rho$ can be exploited for achieving data regarding the structure's health in terms of elastomechanical conditions. In fact, the measurements of $\mathrm{V}$ along proper grids of paths leads to the elaboration of maps of velocity, which allow one to define the level of elastomechanical homogeneity of the investigated structure, emphasizing areas where anomalies are located; moreover, the knowledge of $\mathrm{V}$ values distribution consents to express a qualitative remark on the mechanical effectiveness of the structure, since it is empirically known that the higher the strength the higher the velocity.

Waves velocity measurements are preferentially carried out applying the Direct Transmission Technique (DTT), in which the wave is transmitted by a transducer (Emitter) through the test object and received by a second transducer (Receiver) on the opposite side. This allows measuring the time $\mathrm{T}$ that the wave needs to travel through the object's thickness, from the emitter to the receiver, along a path of length $L$; the average velocity of the wave is simply obtained from the ratio L/T. The DTT is very effective, since the broad direction of wave propagation is perpendicular to the source surface and the signal travels through the entire thickness of the item. Standards concerning the determination of waves velocity in structures, e.g. Europeans EN 12504-4 (EN 12504-4, 2004) and EN 14579 (EN 14579 , 2004), suggest, therefore, the application of this kind of signals transmission.

Nevertheless, there are many kinds of structures, e.g. slabs, retaining walls, piers, in which the DTT cannot be performed, because only one side of the item is accessible. In these cases the Indirect Transmission Technique (ITT), in which both the emitter and the receiver transducers are placed on the same side of the investigated object, or the Semi-direct Transmission Technique (STT), in which transducers are placed on adjacent faces, might be used. Generally speaking, ITT and STT are less effective than the DTT because the amplitude of the received signal is lower, and the pulse propagates in a concrete layer just beneath the surface. These remarks have since now not allowed ITT and STT systematic development, and the scientific literature concerning their use is still quite poor. Despite that, ITT skills of ease to be performed, high potential to evaluate the quality and the characteristics of concrete covering on site, immediacy and low cost, claim to thorough examine its suitability in concrete diagnosis on site, and then to develop studies concerning the standardization of its application. In Annex A of the EN 12504-4 the determination of the pulse velocity via the ITT is illustrated. It is highlighted that there is some uncertainty 
regarding the exact length of the transmission path, since the areas of contact between the transducers and the item are of significant size. It is therefore suggested to make a series of measurements with the transducers at different distances apart to eliminate this uncertainty. The transmitting transducer shall be placed in contact with the item surface at a fixed point $\mathrm{x}$, and the receiving transducer shall be placed at fixed increments $\mathrm{x}_{\mathrm{n}}$ along a chosen line on the surface. The signals transit times recorded should be plotted as points on a graph, showing their relation to the distance separating the transducers. An example of such a plot is shown in Fig. 3, extracted from Annex A. The slope of the best straight line drawn through the points $(\tan \phi)$ shall be measured and recorded as the mean pulse velocity along the chosen line on the concrete surface. Where the points measured and recorded in this way indicate a discontinuity, it is likely that a surface crack or surface layer of inferior quality is present and a velocity measured in such an instance is unreliable.
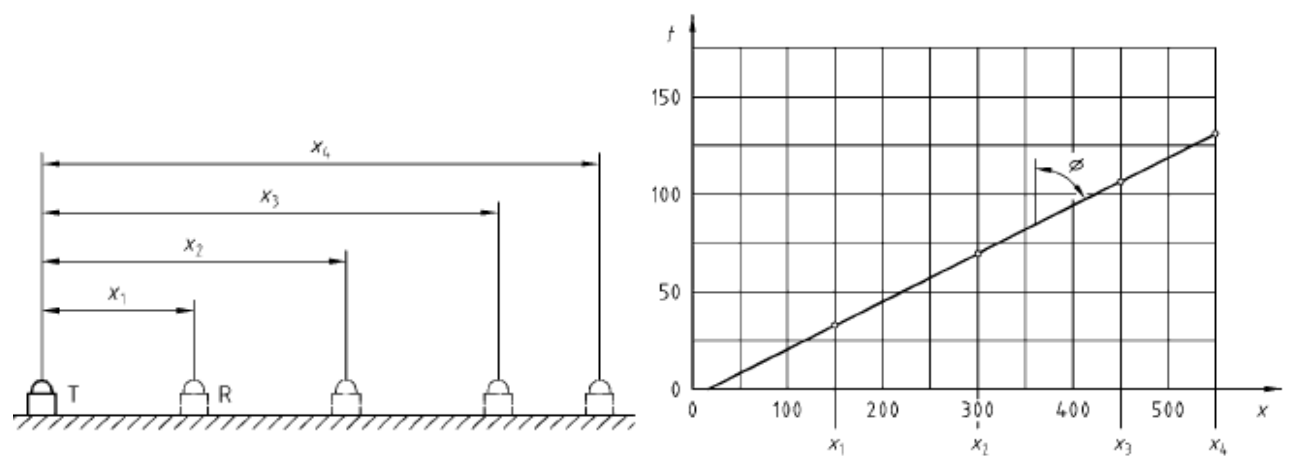

Fig. 3. Example of the determination of pulse velocity by ITT. Extracted from EN 12504-4, 2004 - Annex A

One of the main skills of the ITT is the possibility of cracks depth estimation. An estimate of the depth of a crack visible at the surface can be obtained by measuring the transit times across the crack for two different arrangements of the transducers placed on the surface. One suitable arrangement requires that the transmitting and receiving transducers are placed on opposite sides of the crack and equidistant from it (BS 1881, 1986). Two values of this distance $x$ are chosen, one being twice that of the other, and the transit times corresponding to these are measured. If the first value of $x$ chosen is $x_{1}$ and the second value $x_{2}$ and the transit times corresponding to these are $T_{1}$ and $T_{2}$ respectively, then the crack depth $h$ is:

$$
\mathrm{h}=\mathrm{x}_{1} \sqrt{\frac{4 \mathrm{~T}_{1}^{2}-\mathrm{T}_{2}^{2}}{\mathrm{~T}_{2}^{2}+\mathrm{T}_{1}^{2}}}
$$

Equation (2) is derived by assuming that the plane of the crack is perpendicular to the item surface and that the material in the surrounding area of the crack is of reasonably uniform quality. A check may be made to assess whether the crack is lying in a plane perpendicular to the surface by placing both transducers near to the crack and moving one of them far from the crack. If the transit time decreases, this indicates that the crack slopes towards the direction in which the transducer was moved. It is important that the distance $x$ is measured accurately and that a very good coupling is guaranteed between the transducers and the concrete surface. The method is valid provided the crack is not filled with water. 
Another method of test uses the indirect method where a discontinuity appears in the graph drawn following the indication of the standards (EN 12504-4, 2004; EN 14579, 2004) as previously explained. In this case, if $\mathrm{L}$ is the distance separating the transducers corresponding to which the slope of the line distance-time changes, while $T_{1}$ and $T_{2}$ are the transit times corresponding to this change, then the crack depth $h$ is:

$$
\mathrm{h}=\frac{\mathrm{L}}{2}\left(\frac{\mathrm{T}_{2}}{\mathrm{~T}_{1}}-\frac{\mathrm{T}_{1}}{\mathrm{~T}_{2}}\right)
$$

As previously stated, the direction in which the maximum energy is propagated is at right angles to the face of the transmitting transducer, so that the DTT is the most effective operative procedure. However, the DTT has some limits too. The major limit consists in describing the wave's characteristics field of the object using for each path only one value of that characteristic, i.e., hypothesizing that the average value is homogeneous along each wave path. This assumption prevents from pinpointing the position of the detected anomaly inside the object. A promising way for overcoming this limit is the use of the tomographic technique, which uses numerical analysis as a real measurement instrument, combining the results of several DTT applications for a sharper and reliable investigation of the object.

\subsection{Tomography}

One emerging technique for advanced imaging of materials is Acoustic Tomography (AT). AT uses technology invented for the biomedical field to display the interior of engineered structures. The spatial distribution of acoustic velocity and attenuation are imaged and then correlated with properties directly related to physical conditions (Belanger \& Cawley, 2009; Rhazi, 2006; Leonard Bond et al., 2000; Kepler et al.,2000; Meglis et al.,2005) . The velocity is determined by the elastic properties and density, while the attenuation is determined by the inelastic property of the medium.

\subsubsection{Generalities}

Travel time tomography, a type of AT, represents the natural evolution of the DTT: the signals emitted by different sources are acquired by several receivers arranged so as to allow the taking of a large number of measurements of transit time of signals travelling along pathways at different inclinations which intersect each other on flat sections of the structure. This makes it possible to apply an algebraic system whose unknowns are signal velocity at the nodes of a network arranged on the flat section of the medium containing source and receiver. Thus travel time tomography allows determination of velocity distribution on flat sections of the item being investigated. The method's degree of resolution depends on the distance between sources and receivers, on the measurement step, on angular coverage by means of the trajectories of the studied section. Travel time tomography makes it possible to overcome the major limit of the DTT, that is, the impossibility of discriminating an extended alteration along the source-receiver pathway from an anomaly confined to a part of it only, since for each trajectory joining source and receiver the behavior of the element inside the medium is described by means of a single velocity value. In virtue of the thick net of intersecting trajectories, travel time tomography returns velocity distribution on the section under study with high definition, making it possible to localize precisely and in detail any anomalies that may be present. 
It has long been believed that attenuation is more suitable than velocity (or travel time) to study the inner properties of materials, because an anomaly has a greater effect on the attenuation of a signal than on the propagation time (Best et al., 1994; Hudson, 1981). In fact, as previously mentioned, wave's characteristics such as attenuation, scattering and frequency content may allow one to get relevant information about the material, because of the reliance of the propagation on the properties of the medium through which waves travel. Different materials absorb or attenuate the wave power at different rates, depending on complex interactive effects of material characteristics, such as density, viscosity, homogeneity. Additionally, waves are reflected by boundaries between dissimilar materials, so that changes in materials structure, e.g. presence of discontinuities or defects, can affect amplitude, direction, and frequency content of scattered signals. In this context special credit has to be given to spectral attenuation tomography, which returns the attenuation coefficient distribution on flat sections of the item being investigated.

The main limitations on the AT widespread diffusion are the longer times of execution compared to the traditional operative procedures, the higher cost of the instrumental sets (arrays of sources and receivers, multichannel acquirers), the complexity of the reconstruction of velocity or attenuation distribution starting from signals acquisition.

As previously stated, acoustic investigation methods exploit the transmission and reflection characteristics of mechanical waves with appropriate frequencies passing through the investigated item. Elastic waves propagate in different manner through different solid materials and cavities, thus enabling fault detection. The waves are in most cases generated by a piezoelectric transducer fed with a voltage pulse. The receivers are accelerometers, appropriate positioned based on the measurement type. The tomography represents an improvement to the classic techniques of wave direct transmission, being able to perform tests also on non-perpendicular wave paths. It is so possible to reconstruct a 2D image of the distribution of the wave propagation parameters (e.g. velocity, attenuation) within the analyzed structure, or in one of its sections. These images allow the identification of variations correlated with defects, malformations, cracks etc. Acoustic tomography implies that a ill posed linear equations system has to be solved, in order to determine the distribution of the chosen wave parameter (e.g. velocity, attenuation) in selected sections of the tested structure, thus highlighting the presence of anomalies (Berryman, 1991). Different inversion algorithms are available for determining this distribution starting from signal transmission and acquisition.

\subsubsection{Travel time tomography}

An acoustic wave propagating through any object spends a definite time to travel from a point to another of the object. The wave covers the path between the two points spending the time $t$ and propagating with a mean velocity $V$. When the distance 1 between the two points reduces to zero a local velocity $\mathrm{Vp}$, and a local slowness $\mathrm{s}=1 / \mathrm{Vp}$ can be defined for the point $\mathrm{p}$. In mathematical terms this behavior can be expressed by the following equation:

$$
\int_{\text {path }} \frac{\mathrm{l}}{\mathrm{v}} \mathrm{dl}=\int_{\text {path }} \mathrm{s} \mathrm{dl}=\mathrm{t}
$$

The acoustic behavior of a selected section of the object is then defined when the slowness $\mathrm{s}(\mathrm{x})$ is known continuously in every point $\mathrm{x}$ of the investigated section. This function can be 
approximated dividing the section into a grid of $\mathrm{n}$ rectangular cells (pixels) in which $\mathrm{V}$ is supposed to be constant. The tomographic problem consists in obtaining the slowness of the $\mathrm{n}$ pixels starting from the knowledge of $\mathrm{m}$ travel times $\mathrm{t}$ measured along a series of paths joining couples of transducers located on opposite or adjacent sides of the section. The waves paths depend on the velocity distribution, and their sharp definition is a not easy problem to solve, especially when dealing with structures made of different materials, such as stone masonry, or with a degree of intrinsic non-homogeneity, such as concrete; a valid approximation may be the linear tomography, which considers the paths to be straight. In order to obtain the values of the slowness in the grid, the following equations system, which is the matrix form for equation (4), has to be solved:

$$
\mathrm{PS}=\mathrm{T}
$$

where :

$\mathrm{T}=\left[\mathrm{t}_{1}, \mathrm{t}_{2}, \ldots \mathrm{t}_{\mathrm{m}}\right]$ is the vector of measured travel times;

$\mathrm{S}=\left[\mathrm{s}_{1}, \mathrm{~s}_{2}, \ldots \mathrm{s}_{\mathrm{n}}\right]$ is the vector of slowness;

$\mathrm{P}=\left[\mathrm{l}_{11}, \mathrm{l}_{12}, \ldots \mathrm{l}_{\mathrm{nm}}\right]$ is the coefficients matrix, whose generic element $\mathrm{l}_{\mathrm{ij}}$ is the length of the $\mathrm{i}^{\text {th }}$ path in the $\mathrm{j}^{\text {th }}$ cell.

Thus, the tomographic solution consists in determining the vector $S$ as:

$$
\mathrm{S}=\mathrm{P}_{\mathrm{g}}^{-1} \mathrm{~T}
$$

To avoid instability in matrix inversion, the number $\mathrm{n}$ of cells must be smaller than the number $\mathrm{m}$ of measured travel times. If the inverse of $\mathrm{P}$ exists it can be directly evaluated. However, the inverse of $\mathrm{P}$ generally does not exist since $\mathrm{P}$ is not a square matrix, it is ill conditioned, and it has not full rank. Thus, other methods, such as iterative ones, have to be used to solve the problem.

\subsubsection{Spectral attenuation tomography}

As previously stated, the propagation velocity, although a significant parameter, limits the investigation at the analysis of propagation times, not taking into consideration important information regarding the way the waves are propagating. When a signal passes through a specific item or structure, certain frequency components of this signal are altered, the item behaving like a filter performing modifications on the frequency components' magnitude and phase. Therefore, it would be reasonable to approach the tomographic problem also in terms of frequency spectrum changes and not only in terms of propagation times. This type of approach is documented by various studies performed in the geophysics environment, and furthermore in the approach of Quan and Harris (Quan \& Harris, 1997), based on the observation that the frequency attenuation increments with the frequency of the signal, meaning that the higher frequencies of a signal are more rapidly attenuated than the lower ones. The main advantage of the frequency analysis is the immunity against disturbing factors such as spherical divergence, reflection and transmission effects and coupling of the receiver with the transmitter, which can affect the correct interpretation of the received signals.

For the purpose of estimating attenuation, the process of waves propagation can be assumed as described by linear system theory. If the amplitude spectrum of an incident wave is $S(f)$ and the instrument-medium response is $\mathrm{G}(\mathrm{f}) \cdot \mathrm{H}(\mathrm{f})$, then the received amplitude spectrum $\mathrm{R}(\mathrm{f})$ may be, in general, expressed as : 


$$
R(f)=G(f) \cdot H(f) \cdot S(f)
$$

where the factor $\mathrm{G}(\mathrm{f})$ includes geometrical spreading, instrument response, source-receiver coupling, radiation patterns, and reflection-transmission coefficients, and the phase accumulation caused by propagation, and $\mathrm{H}(\mathrm{f})$ describes the attenuation effect on the amplitude. It can be assumed that the effects included in factor $G(f)$ are not frequency dependent, thus it can be simplified as $G(f)=G$. In structural diagnosis, the $H(f)$ factor is of greater interest. Experiments indicate that attenuation is usually proportional to frequency (Johnston, 1981), that is, response H(f) may be expressed as:

$$
H(f)=\exp \left(-f \int_{\text {path }} \alpha_{0} \mathrm{dl}\right)
$$

where the integral is taken along the supposed straight wavepath, and $\alpha_{0}$ can be regarded as an intrinsic attenuation coefficient. The tomography's goal is to estimate the medium response $H(f)$, or more specifically, the attenuation coefficient $\alpha_{0}$, from knowledge of the input spectrum $S(f)$ and the output spectrum $R(f)$. A direct approach is to solve equation (8) by taking the logarithm and obtaining:

$$
\int_{\text {path }} \alpha_{0} \mathrm{dl}=\frac{1}{\mathrm{f}} \ln \left[\mathrm{G} \frac{\mathrm{S}(\mathrm{f})}{\mathrm{R}(\mathrm{f})}\right]
$$

Equation (9) may be used to estimate the integrated attenuation at each frequency and is called the amplitude decay method. However, as described above, the factor G lumps many complicated processes together, and is very difficult to be determined. Furthermore, the calculation of attenuation based on individual frequencies is not robust because of poor individual signal-to-noise.

To overcome these difficulties, Quan and Harris (Quan \& Harris, 1997) developed a statistically based method that estimates the attenuation coefficient $\alpha_{0}$ from the spectral centroid downshift over a range of frequencies. An analog relationship to that between signal velocity along the wavepath and travel time connects the attenuation to the difference of the signals' spectrum frequency centroid, the latter being a parameter indicating the center of the signals' distribution in frequency. As mentioned, during wave propagation the higher frequencies are attenuated more rapidly than the lower frequency components, downshifting the centroid towards the lower frequencies. The centroid frequency of the input signal $\mathrm{S}(\mathrm{f})$ is defined as:

$$
f_{S}=\frac{\int_{0}^{\infty} f S(f) d f}{\int_{0}^{\infty} S(f) d f}
$$

and the variance is:

$$
\sigma_{\mathrm{S} 2}=\frac{\int_{0}^{\infty}\left(\mathrm{f}-\mathrm{f}_{\mathrm{S}}\right)^{2} \mathrm{~S}(\mathrm{f}) \mathrm{df}}{\int_{0}^{\infty} \mathrm{S}(\mathrm{f}) \mathrm{df}}
$$

Similarly, the centroid frequency of the received signal $R(f)$ is:

$$
f_{R}=\frac{\int_{0}^{\infty} f R(f) d f}{\int_{0}^{\infty} R(f) d f}
$$

and its variance is: 


$$
\sigma_{\mathrm{R} 2}=\frac{\int_{0}^{\infty}\left(\mathrm{f}-\mathrm{f}_{\mathrm{R}}\right)^{2} \mathrm{R}(\mathrm{f}) \mathrm{df}}{\int_{0}^{\infty} \mathrm{R}(\mathrm{f}) \mathrm{df}}
$$

where $R(f)$ is given by equation (7). If the factor $G$ is independent on the frequency $f$, then $f_{R}$ and $\sigma_{R}^{2}$ will be independent on $G$. This is a major advantage of using the spectral centroid and variance rather than the actual amplitudes. For the special case where the incident spectrum S(f) is Gaussian, assuming a linear-dependence model of attenuation to frequency, the attenuation coefficient $\alpha_{0}$ can be estimated as follows:

$$
\int_{\text {path }} \alpha_{0} \mathrm{dl}=\frac{\mathrm{f}_{\mathrm{S}}-\mathrm{f}_{\mathrm{R}}}{\sigma_{\mathrm{S} 2}}
$$

where $f_{S}$ and $f_{R}$ are the centroid frequency for the source and receiver, respectively, and $\sigma_{S}$ is the variance, or bandwidth, of the source signal. The previous relationship states that the attenuation is proportional to the centroid frequency difference which has downshifted from the original source centroid $f_{S}$, to the centroid of the received signal $f_{R}$. The total amount of centroid frequency downshift depends on the attenuation characteristics along the acoustic path. The tomographic formula relating frequency shift with the attenuation projection is exact only for Gaussian spectra. Yet, similar derivations can also be obtained for other frequency compositions, which implies that the estimates of relative attenuation are not sensitive to small changes in spectrum shapes, and points out the robustness of this method.

It is worth noting that equation (14) is in the same form as (4), with the intrinsic attenuation coefficient $\alpha_{0}$ in (14) corresponding to the slowness $1 / \mathrm{V}$ in (4). The expression of frequency centroid difference in (14) corresponds to the travel time $t$ in (4). This similarity makes the attenuation tomographic inversion easy to conduct applying the same algorithms developed for travel time tomography, simply replacing $1 / \mathrm{V}$ with $\alpha_{0}$ and $\mathrm{t}$ with $\left(\mathrm{f}_{\mathrm{S}}-\mathrm{f}_{\mathrm{R}}\right) / \sigma_{\mathrm{S}}{ }^{2}$.

As stated above, equation (14) is the basic formula for spectral attenuation tomography. It can be written also in a discrete form as:

$$
\sum_{\mathrm{j}=1}^{\mathrm{N}} \mathrm{l}_{\mathrm{ij}} \alpha_{0 \mathrm{j}}=\frac{\mathrm{f}_{\mathrm{Si}}-\mathrm{f}_{\mathrm{Ri}}}{\sigma_{\mathrm{Si}}^{2}} \quad(\mathrm{i}=1, . . \mathrm{M})
$$

where $i$ represents the $i^{\text {th }}$ path, $j$ the $j^{\text {th }}$ parameterized cell of the medium, and $l_{i j}$ is the length of the $\mathrm{i}^{\text {th }}$ path in the $\mathrm{j}^{\text {th }}$ cell (Fig. 4).

The previous equations system can be written in matrix form as:

$$
\mathrm{L} A=\mathrm{F}
$$

where :

$\mathrm{F}=\left[\mathrm{F}_{1}, \mathrm{~F}_{2}, \ldots \mathrm{F}_{\mathrm{m}}\right]$ is the vector of calculated centroid frequency downshift, in which $\mathrm{F}_{\mathrm{i}}=\left(\mathrm{f}_{\mathrm{Si}}-\right.$ $\left.\mathrm{f}_{\mathrm{Ri}}\right) / \sigma_{\mathrm{Si}}^{2}$ has been assumed;

$A=\left[\alpha_{01}, \alpha_{02}, \ldots \alpha_{0 n}\right]$ is the vector of attenuation coefficients;

$\mathrm{L}=\left[\mathrm{l}_{11}, \mathrm{l}_{12}, \ldots \mathrm{l}_{\mathrm{nm}}\right]$ is the coefficients matrix, whose generic element $\mathrm{l}_{\mathrm{ij}}$ is the length of the $\mathrm{i}^{\text {th }}$ straight path in the $\mathrm{j}^{\text {th }}$ cell.

The intrinsic attenuation coefficient $\alpha_{0}$ is in the unit of [ $\left.\mathrm{dBsm}^{-1}\right]$.

Moreover, the attenuation coefficient $\alpha_{0}$ can be also expressed as: 


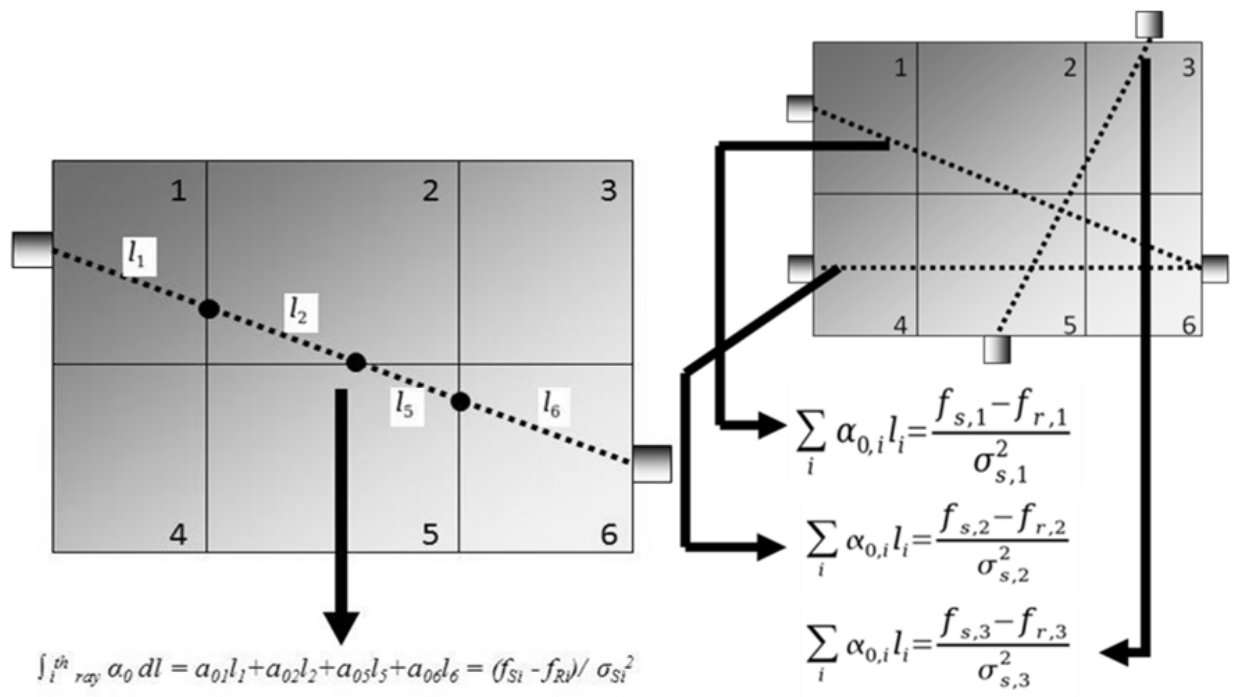

Fig. 4. Example of spectral attenuation tomography system equation

$$
\alpha_{0}=\frac{\pi}{\mathrm{QV}}
$$

with $\mathrm{Q}$ the quality factor of the material and $\mathrm{V}$ the propagation velocity. In wave propagation problems the $Q$ factor is useful for characterizing wave attenuation, being defined as the ratio of the total kinetic energy and energy loss in one vibration cycle (Sheriff \& Geldart, 1995; Knopoff, 1964). Geophysicists and seismologists often use the Q factor to study this attenuation in rocks. An infinite $Q$ means that there is no attenuation. This factor is a function of the mineral composition of rocks as well as of their mechanical performances (Ilyas, 2010). Numerous field observations have demonstrated that the quality factor $Q$ appears to be a constant over a large frequency range in the signal bandwidth. This is widely accepted in the geophysics community and is referred to as the constant $Q$ model. Hence, the tomographic inversion can be applied for the case of the spectral attenuation in a similar way as for the arrival times and the propagation velocity. The model obtained for $Q$ is consistent with the one obtained for the velocity; therefore the information regarding the velocity distribution can be used for calculating $Q$ itself.

\subsubsection{Resolution algorithms}

A common method to obtain the solution of equations system (5) in the least square sense is the Singular Value Decomposition (SVD) (Berryman, 1991; Herman, 1980; Ivansson, 1986). The SVD can be used for computing the pseudo-inverse of the coefficient matrix P. Indeed, this method produces a diagonal matrix $\mathrm{D}$, of the same dimension of $\mathrm{P}$ and with nonnegative diagonal elements in decreasing order, and two unitary matrices $U$ and $V$ so that $P$ $=\mathrm{UDV}^{\mathrm{T}}$. Then, the pseudo-inverse of the matrix $\mathrm{P}$ with singular value decomposition is $\mathrm{P}^{+}=$ $\mathrm{VD}^{-1} \mathrm{UT}^{\mathrm{T}}$ and the solution of equations system (5) can be written as $\mathrm{S}=\mathrm{P}^{+} \mathrm{T}$. The same result can be assumed for the spectral attenuation tomography, simply considering system (16) instead of system (5). This solution is the minimum norm solution and it is only 
mathematically suitable. The inverse problem is ill posed and ill conditioned, making the solution sensitive to measurement errors and noise. Regularization methods are needed to treat this ill posedness. It can be shown that the small singular values mainly represents the noise and can be discarded. Truncated SVD (SVDT) may be considered as having a filter, and hence it is less sensitive to high frequency noise in the measurements.

Because of the non linear relationship velocity - travel time and attenuation coefficient centroid frequency downshift, it is almost impossible to find the solution for systems (5) and (16) by a single step algorithm using a linear approximation. Thus, iterative methods (Gilbert, 1972; Lo \& Inderwiesen, 1994) can be used, such as Algebraic Reconstruction Technique (ART) (Gordon, 1974; Gordon et al., 1970) and Simultaneous Iteration Reconstruction Technique (SIRT) (Dines \& Lytle, 1979; Lakshiminarayanan \& Lent, 1979; Jansen et al., 1991). Both methods need a starting value of velocity or attenuation, and then they modify iteratively this value by minimizing the difference between the measured travel time or centroid frequency downshift and the value calculated in the previous iteration. While ART goes on wavepath after wavepath, SIRT takes into account the effect of all wavepaths crossing each cell. In the n-dimensional space each equation in (5) and (16) represents a hyperplane. When a unique solution exists, the intersection of all the hyperplanes is a single point. A computational procedure to locate the solution consists in starting with an initial solution, denoted by:

$$
\mathrm{q}^{(0)}=\left(\mathrm{q}_{1}^{(0)}, \mathrm{q}_{2}^{(0)}, \ldots, \mathrm{q}_{\mathrm{n}}^{(0)}\right)
$$

where q signifies velocity or attenuation coefficient depending on whether travel time or spectral attenuation tomography is being performed. This initial solution is projected on the hyperplane represented by the first equation in (5) or (16) giving $\mathrm{q}^{(1)}$. This value is then projected on the hyperplane represented by the second equation in (5) or (16) to yield $\mathrm{q}^{(\mathrm{i}-1)}$ and so on. When $\mathrm{q}^{(\mathrm{i}-1)}$ is projected on the hyperplane represented by the $\mathrm{i}^{\text {th }}$ equation to yield $\mathrm{q}^{(\mathrm{i})}$, the process can be mathematically described by:

$$
\mathrm{q}^{(\mathrm{i})}=\mathrm{q}^{(\mathrm{i}-1)}+\frac{\mathrm{b}_{\mathrm{i}-\mathrm{l}_{\mathrm{i}}^{\mathrm{T}} \mathrm{q}^{\mathrm{i}-1}}}{\mathrm{l}_{\mathrm{i}}^{\mathrm{T}} \mathrm{l}_{\mathrm{i}}} \mathrm{l}_{\mathrm{i}}
$$

where $l_{i}$ is the $i^{\text {th }}$ raw of the matrix $L$ and $b_{i}$ represents $t_{i}$ or $F_{i}$ depending on whether travel time or spectral attenuation tomography is being performed. A single iteration of ART is completed when each row of $\mathrm{P}$ has been cycled.

For an over determined problem, $m>n$, ART does not give a unique solution, but this depends on the starting point. The tomographic system is normally over determined and measurement noise is present. In this case a unique solution does not exist and the solution found by ART will oscillate in the neighborhood of the intersections of the hyperplanes. The SIRT algorithm uses the same equations as in the ART algorithm; the difference is that SIRT modifies the attenuation model taking into account at each iteration the effect of all wave paths crossing each cell. The new value of each cell is the average value of all the computed values for each hyperplane:

$$
q_{j}^{(k+1)}=q_{j}^{(k)}+N_{j j}^{-1} \sum_{i=1}^{m} \frac{\left(b_{i}-\sum_{w=1}^{n} l_{i w} q_{w}^{(k)}\right) l_{i j}}{\sum_{r=1}^{m} l_{i r}^{2}}
$$


Then, using the SIRT algorithm better solutions are usually obtained at the expense of slower convergence.

\subsection{Impact echo method}

Another development of the classic operative procedures is the impact-echo model, which represents in some way an evolution in the ITT. This method is based on the principle of reflection: a signal propagating in a medium is reflected on encountering an anomaly of any kind inside it (Sansalone \& Streett, 1997; Carino, 2001). On striking the medium with a succession of impulses, usually generated by mechanical impact with a hammer, these are reflected from the interface between the medium and the air if the piece is homogeneous or by the defect if the medium presents an interruption in continuity; in this case the reflection caused by the limiting surface is addressed as base echo, while the reflection caused by any imperfections there may be in the medium is addressed simply as echo. By arranging source and receiver on the surface of the medium it is possible to visualize with suitable instrumentation the echoes generated by the reflection of the signal in the medium; this makes it possible to establish the kind of reflection it is, that is, if it is caused by limit surfaces or something else, and to locate the obstacle inside the medium as a function of amplitude and the reciprocal position of the signals corresponding to the echoes. The extraction of information usually takes place in the frequency domain.

\subsection{Measurements sets}

The basic instrumental set for the performance of sonic and ultrasonic tests is composed of a source, a receiver and a data acquisition and processing unit, often an oscilloscope and PC. The fundamental difference lies in the characteristics of the source: the sonic method calls for the use of signals containing high energy and thus characterized by low frequency for which the excitation of the material is usually performed with the impact of an instrumented hammer; the ultrasonic method requires the use of high-frequency signals since no excessive dissipation of energy inside the material is foreseen, so the signal is introduced by means of a transducer, usually piezoelectric. Fig. 5 shows the schematic of instrumental sets commonly used in the two kinds of tests.

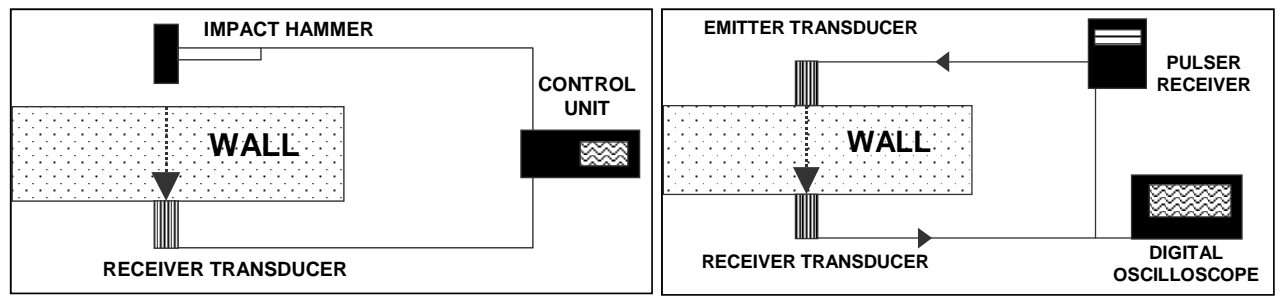

Fig. 5. Instrumentation set up. Sonic Testing (left) and Ultrasonic Testing (right)

When applying the Acoustic Emission procedure, the signal associated to the acoustic emission can be captured by a suitable instrumental setup, usually composed of highfrequency transducers, amplifiers, filters, systems of acquisition and storage such as digital oscilloscopes or acquisition cards, data processing software and so on, as shown in Fig. 6. 


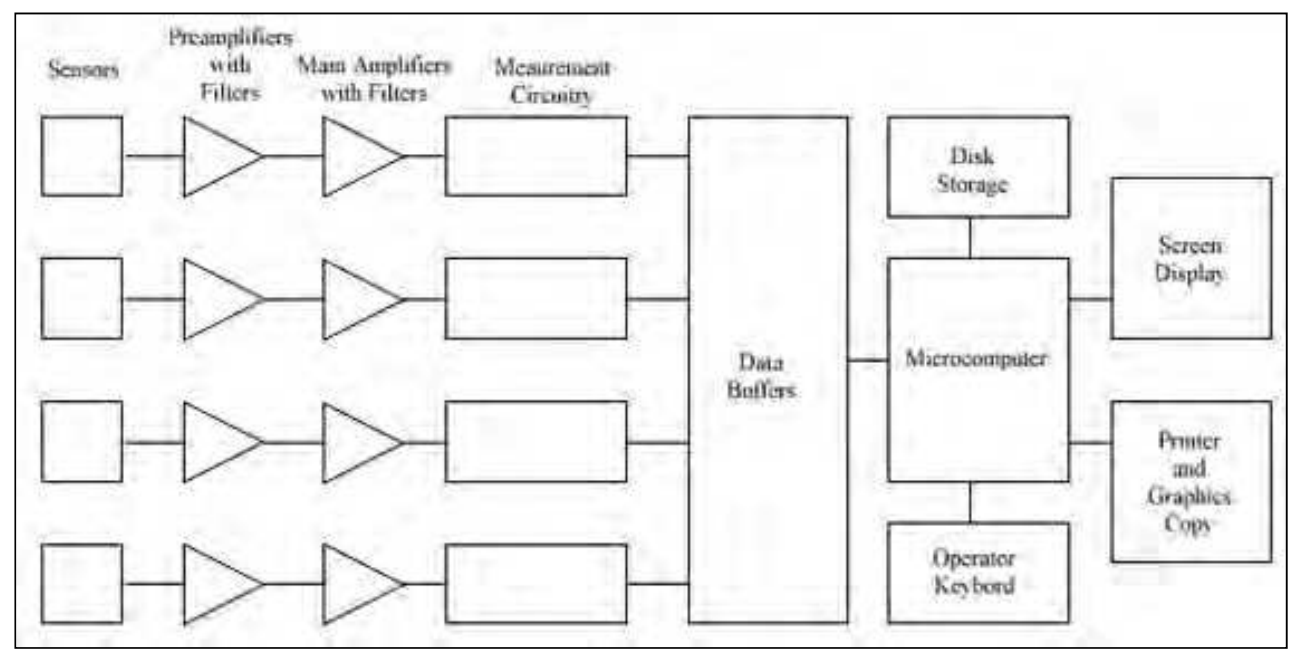

Fig. 6. Schematic diagram of a basic four-channel Acoustic Emission testing system. Extracted from NDT Education Resource Center

Impact-echo testing relies on three basic components, as shown in Fig. 7:

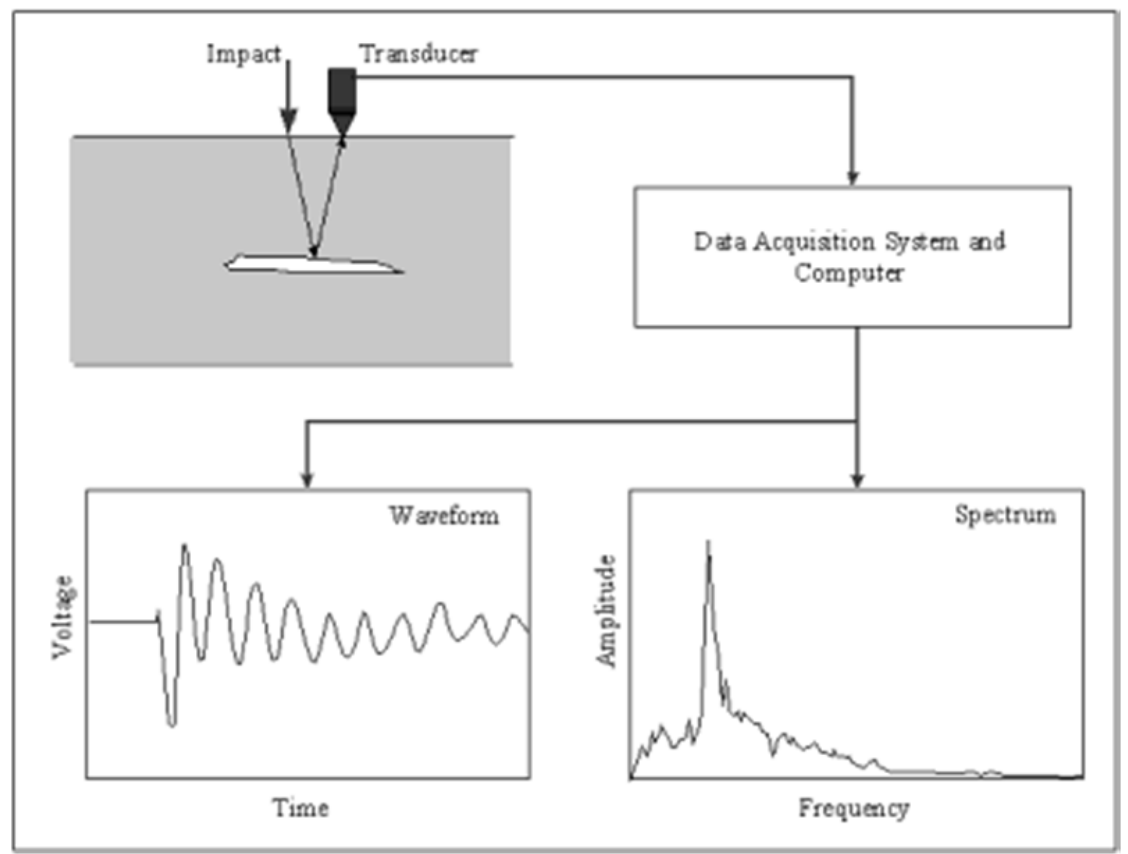

Fig. 7. Schematic diagram of the Impact-Echo test. Extracted from Sansalone \& Streett, 1997 
- a mechanical impactor capable of producing short-duration impacts, the duration of which can be varied;

- a high-fidelity receiver to measure the surface response;

- a data acquisition-signal analysis system to capture, process, and store the waveforms of surface motion.

\section{Case study}

In order to deepen the reliability of acoustic methods in buildings faults detecting and materials characterization, an experimental program has been started, experimenting both DTT and AT approaches to a full scale masonry model.

\subsection{Materials}

The two operative procedures - DTT and AT - have been carried out on a full scale real stone masonry expressly made by the Lab of Structural Engineering Dept. The wall is $0.90 \mathrm{~m}$ wide, $0.62 \mathrm{~m}$ high and $0.38 \mathrm{~m}$ thick, and it is made of Trachite blocks sized $0.20 \mathrm{~m} \times 0.38$ $\mathrm{m} \times 0.12 \mathrm{~m}$, settled as shown in Fig. 8 and jointed with cement lime mortar. The block assigned to the central position of the wall was not settled, thus realising a macro-cavity with the same size of the missing block, and assumed as a known anomaly. Mortar joints have been assumed to be $1 \mathrm{~cm}$ thick, but since the wall was manually built by a builder, actual dimensions are not so precise.
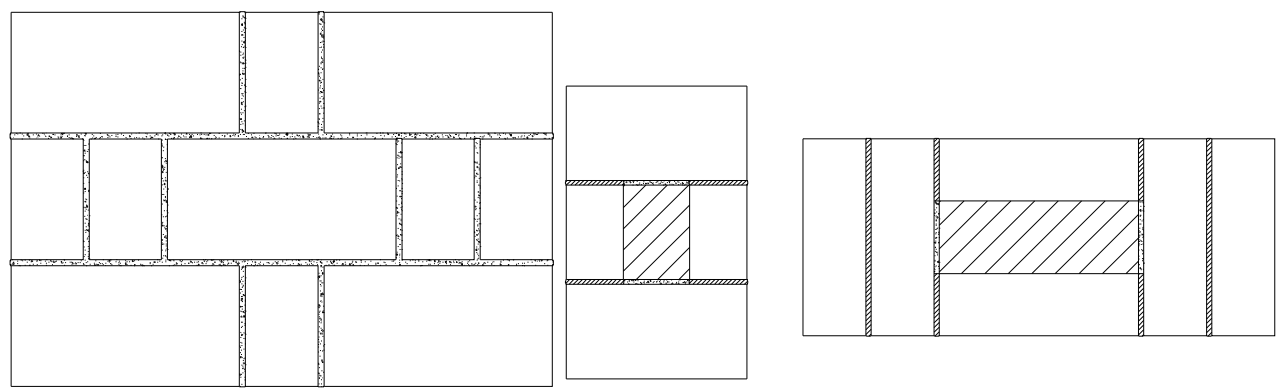

Fig. 8. The full scale real masonry. From left to right: front view, vertical section, horizontal tomographic section

Trachite specimens have been prepared and then tested for the determination of compressive strength and elastic modulus, following the Italian Standards UNI EN 1926, 2000 (UNI EN 1926, 2000) and UNI EN 14580, 2005 (UNI EN 14580, 2005) respectively. Results are shown in Table 1.

\begin{tabular}{|l|l|}
\hline Properties & Value (MPa) \\
\hline Compressive strength & 40.5 \\
\hline Static elastic modulus & 6100 \\
\hline
\end{tabular}

Table 1. Materials properties 


\subsection{Methods}

\subsubsection{Direct transmission technique}

As previously stated, when using the DTT the acoustic signal is transmitted through the test object and received by a second transducer on the opposite side of the structure. Changes in received signal provide indications of variations in material continuity. In this case study 220 emitters and 220 receivers have been arranged in a grid of $11 \times 20$ nodes in the opposite surfaces of the wall (Fig. 9).

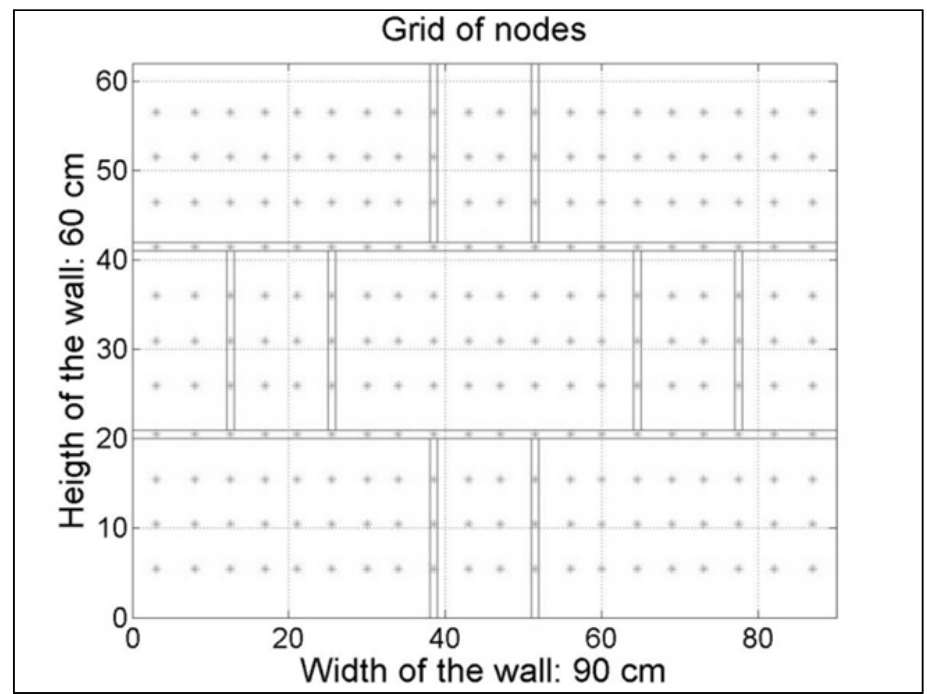

Fig. 9. Grid of test points

At the end of the experimental sessions, 220 signals have been obtained, one for each point of the grid of receivers. From each node of the grid, two parameters - velocity and signal power - have been extracted, in order to investigate the presence of anomalies and obtain some information on the material. The signal power is defined as:

$$
\mathrm{P}_{\text {Total }}=\frac{\int_{0}^{\mathrm{T}}|\mathrm{x}(\mathrm{t})|^{2} \mathrm{dt}}{\mathrm{T}}
$$

where $\mathrm{T}$ is the time duration of the received signal $\mathrm{x}(\mathrm{t})$.

The excitation wave is an acceleration signal. It is a seven and half-cycle tone burst enclosed in a Hanning window, expressed by the following equation (22) and shown in Fig. 10. This waveform has been chosen to reduce the leakage phenomena. The parameter $\mathrm{f}=60 \mathrm{kHz}$ is the characteristic frequency of the emitting transducer.

$$
y(t)=\frac{1}{2} \sin (2 \pi f t)\left[1-\cos \left(\frac{2}{15} 2 \pi f t\right)\right]
$$

The emitter and receiver are piezoelectric transducers with a frequency of $60 \mathrm{kHz}$. The emitter is connected to a signal generator PCG10 Velleman Instruments ${ }^{\circledR}$. Both transducers are connected to a digital oscilloscope interfaced with a laptop. Due to the strong 


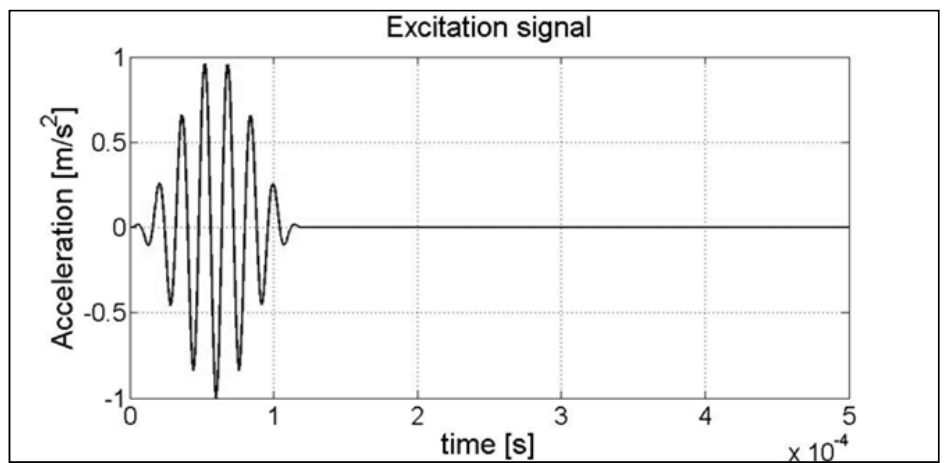

Fig. 10. Excitation signal used in the DTT

attenuation of the transmitted signal, the peak to peak voltage of the generator has been amplified from $13 \mathrm{~V}$ to $39 \mathrm{~V}$ using a transformer between the signal generator and the emitter. The transformer has a transformation ratio of $1 / 3$ and a frequency band of (10-200) $\mathrm{kHz}$. Moreover, an amplifier has been connected between the receiver and the oscilloscope. It has gain equal to 100 or 200, a frequency band of $(10-200) \mathrm{kHz}$ and input impedance of $1 \mathrm{M} \Omega$. The experimental setup is shown in Fig. 11.

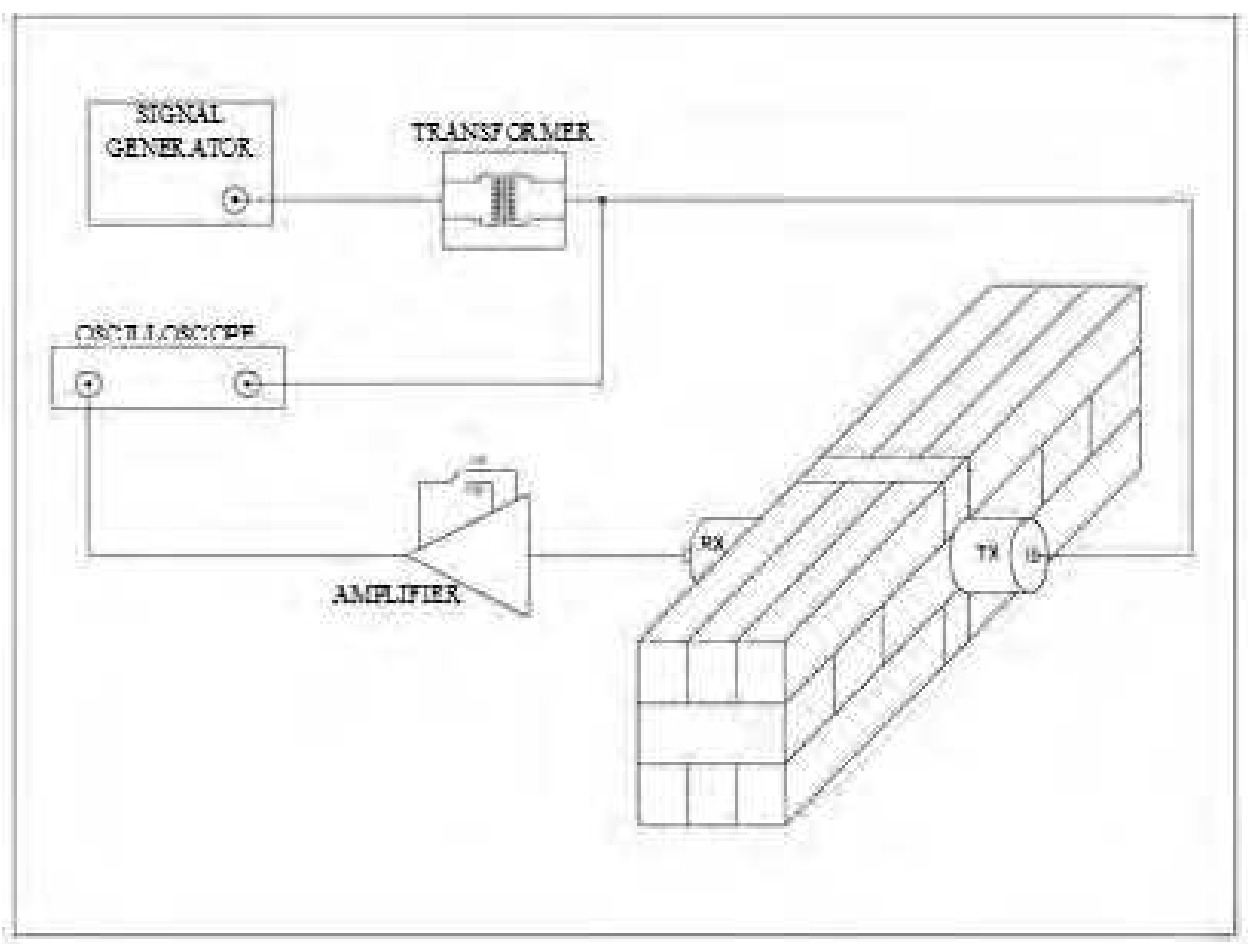

Fig. 11. Experimental setup for DTT application 


\subsubsection{Acoustic tomography}

Aim of the experimental program was also to point out the reliability of both travel time and spectral attenuation AT in detecting building structures faults. With this purpose, the algebraic problem of the tomography inversion has been deepened, the SIRT algorithm has been selected and then numerically developed. After that, the solving algorithm has been implemented in an automated procedure that allows the user to easily obtain a map of the distribution of acoustic parameters (velocity and attenuation) in the selected section of the item. The AT has been applied to a horizontal plane section crossing the wall in order to intercept the central void (Fig. 8). The investigated section was thus $0.90 \mathrm{~m}$ wide and $0.38 \mathrm{~m}$ thick, and it has been divided in 40 cells $0.09 \mathrm{~m} \times 0.095 \mathrm{~m}$. By using this measurements configuration, the section is crossed by 138 paths, and its coverage by the wave paths is excellent. For each path the quantity $\left(f_{S}-f_{R}\right) / \sigma_{S}^{2}$ has been calculated. Thus, systems (5) and (16) consist in 138 equations and 40 unknowns, so as the two equations systems are satisfactorily over determined.

Three kinds of signal have been evaluated in the experimental setup: pulse, sweep and chirp signal. The short voltage pulse $\mathrm{p}(\mathrm{t})$, defined as:

$$
\mathrm{p}(\mathrm{t})=\operatorname{Arect}\left(\frac{\mathrm{t}}{\mathrm{T}}\right)
$$

where $\mathrm{T}$ is the pulse duration and A the amplitude, is generally preferred for estimating the travel time of elastic waves because it involves high power; on the other hand, when using this signal only a poor control on the signal spectrum is usually achievable, thus making the spectral tomography hardly possible. Because of the huge importance of the signal to noise ratio for FFT spectral analysis, a broadband sweep signal has been preferred as source signal. This signal, expressed by the following:

$$
s(t)=\operatorname{Acos}\left(2 \pi f_{0} t+\frac{k}{2} t\right)
$$

where $T$ is the pulse duration, $f_{0}$ is the lower bound of the frequency bandwidth which increases with $\mathrm{k}=2 \pi \Delta \mathrm{f} / \mathrm{T}$ rate and $\mathrm{A}$ is the amplitude, shows a linear relationship between time and frequency. The purpose of using this signal was to extend the frequencies involved in measurements up to $300 \mathrm{kHz}$, aiming at increasing the analysis resolution and at estimating the attenuation coefficient more accurately in a wider signal band. Indeed, the evaluation of the spectral attenuation coefficient requires a spread spectrum of both transmitted and received frequencies in order to accurately estimate both centroid frequency and variance values. Finally, the feasibility of using a chirp signal, described by the equation:

$$
s(t)=A \cos \left(2 \pi f_{0} t+\frac{k}{2} t^{2}\right) \operatorname{rect}\left(\frac{t}{T}\right)
$$

has been evaluated. This kind of signal allowed the performing of received signal spectrum analysis similar to that achievable using the sweep one; moreover, it allowed crosscorrelating the received signal with the source one for travel time estimating, so as to perform an effective travel time tomography.

Based on the requirements posed by the mathematical background presented in the previous sections, an innovative measurements system was set-up according to the diagram presented in Fig. 12. 


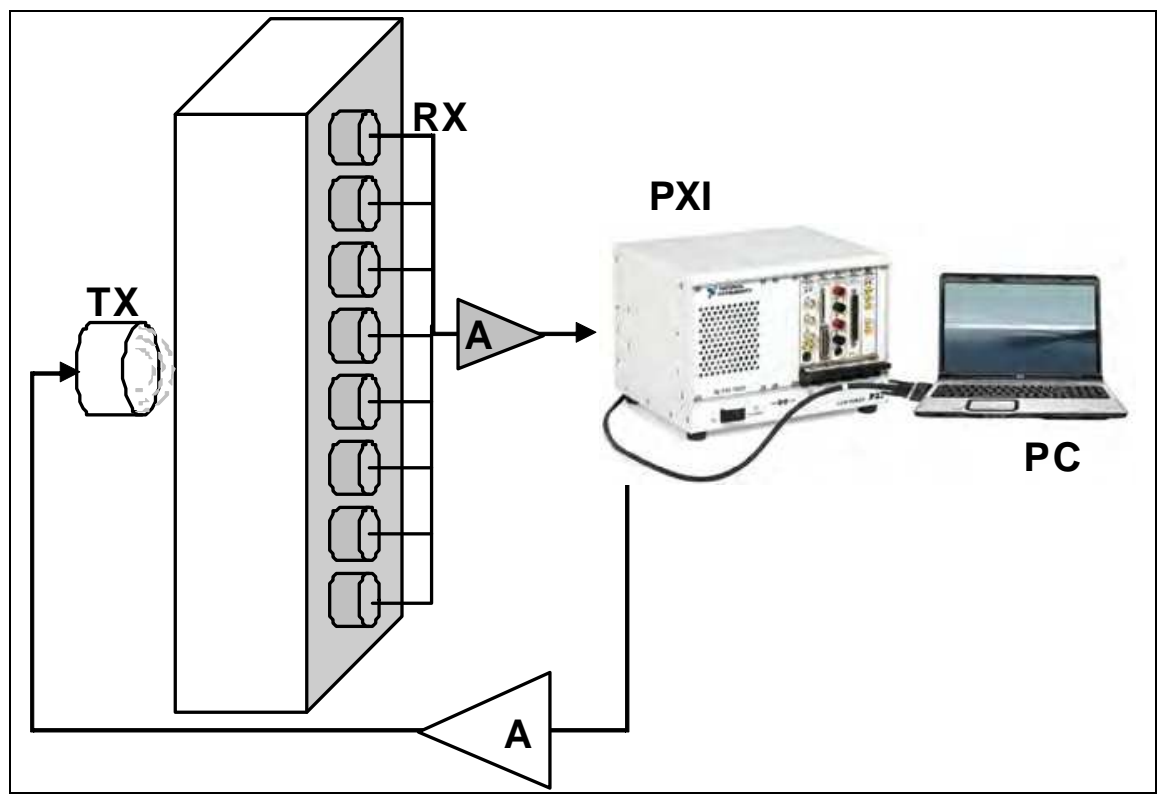

Fig. 12. Set-up of the measurement system

In order to simplify the measurement process, a multi-receiver solution was adopted, using eight accelerometers as receivers for the generated wave. More detailed, the instrumentation setup is composed of the following elements:

- broadband piezoelectric transducer GRW350-D50 Ultran ${ }^{\circledR}$ used as an ultrasonic wave generator. The transducer has diameter of $520 \mathrm{~mm}$, a central frequency of $370 \mathrm{kHz}$ and is fed with a high voltage signal (200 Vpp) for overcoming the high impedance of the analyzed materials;

- $\quad$ eight VS-150-M Vallen ${ }^{\circledR}$ piezoelectric sensors (accelerometers) with a good frequency response in the band of interest $(100-500 \mathrm{kHz})$. Each of these sensors is coupled with its own preamplifier with a gain of $40 \mathrm{~dB}$, thus ensuring an elevated sensibility;

- data acquisition system National Instruments ${ }^{\circledR}$ PXI DAQ with two PXIe-6124 boards having a total of 8 analog inputs and 2 analog outputs. The inputs have a sampling rate of $4 \mathrm{MS} / \mathrm{s}$ each, at 16-bit resolution. The PXI rack is connected through a PCI-Express card to a laptop computer that commands the entire measuring process.

The interface between the PXI data acquisition rack and the computer is based on the virtual instruments created in the LabView ${ }^{\circledR}$ environment, used both for acquiring the output of the eight reception sensors as for generating the sweep signal for the piezoelectric transducer. This signal is generated on one of the analog outputs of the acquisition board and is subsequently amplified using a custom-build amplifier.

The entire measuring process is encapsulated in a user-friendly application that guides the user by the means of a step-by-step procedure. At the beginning of the procedure, the physical parameters of the structure can be specified, allowing the application to calculate the number and position of the measurement points on the surface of the structure at each 
step of the measure. Then the application triggers the generation of the signal and acquires the output of the accelerometers for each measurement step. At the end of the cycle, the tomographic algorithm previously mentioned is applied on the entire set of acquired data in order to produce the tomographic map of the analyzed section.

\subsection{Results}

Results of both DTT and AT operative procedures have been displayed in terms of distribution maps, in order to facilitate data interpretation and anomalies identification. The maps are represented by a 256-levels gray-scale diagram, where the lowest level corresponds to the white color, and the highest level corresponds to the black color. The 256 levels are normalized with respect to the range of the parameter values measured in the represented map.

The map of the distribution of both signal velocity (Fig. 13) and signal power (Fig. 14) has been derived interpolating the data recorded in the grid's nodes (Cannas et al., 2008). Each maps emphasizes areas where anomalies are located, according to feature's specific diagnostic skill. In the center of both the maps the presence of the cavity region can be clearly seen, although it is smaller than expected. The distribution of propagation velocity in the rest of the map seems to be quite uniform, thus confirming that this parameter can easily detect macroscopic anomalies, while it seems to be less affected by minor materials discontinuity such as the mortar joints. The distribution of the signal power, on the other hand, allows to better distinguishing the cavity in terms of both position and extension, and points out that the various interfaces inside the structure affect this feature much more than the velocity.

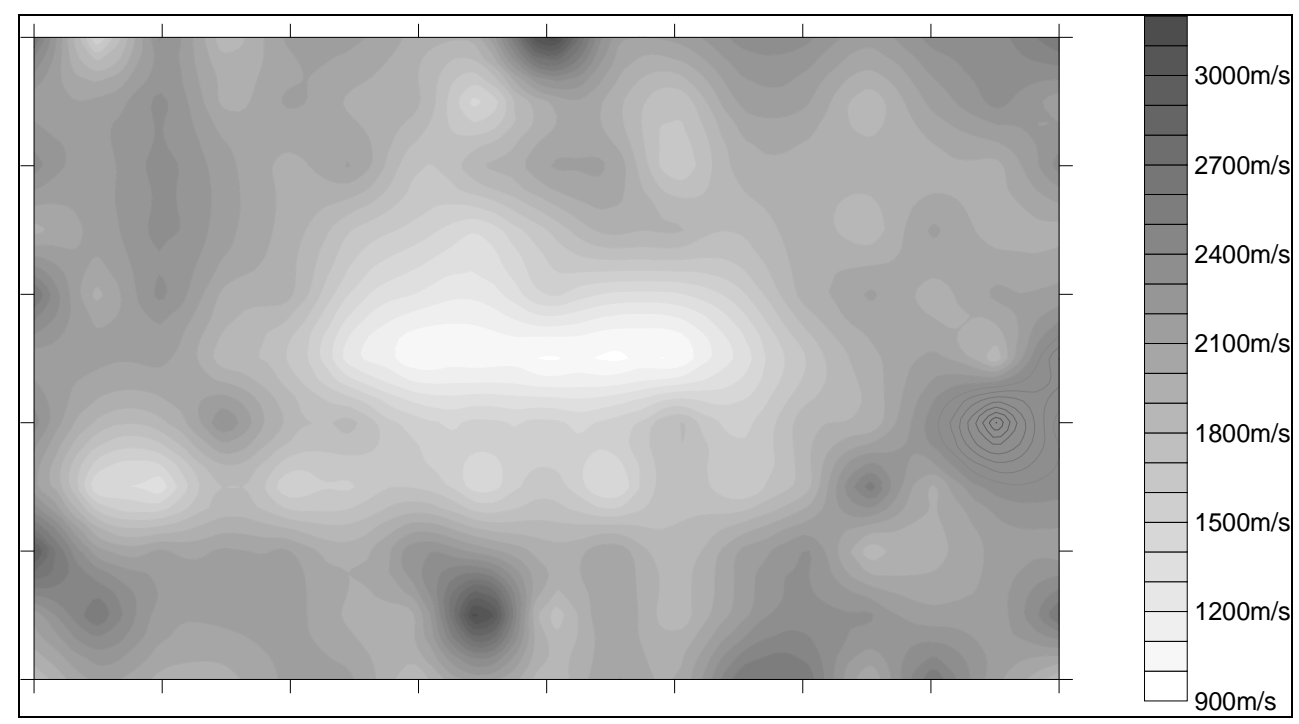

Fig. 13. Propagation velocity $[\mathrm{m} / \mathrm{s}]$ : distribution map. 


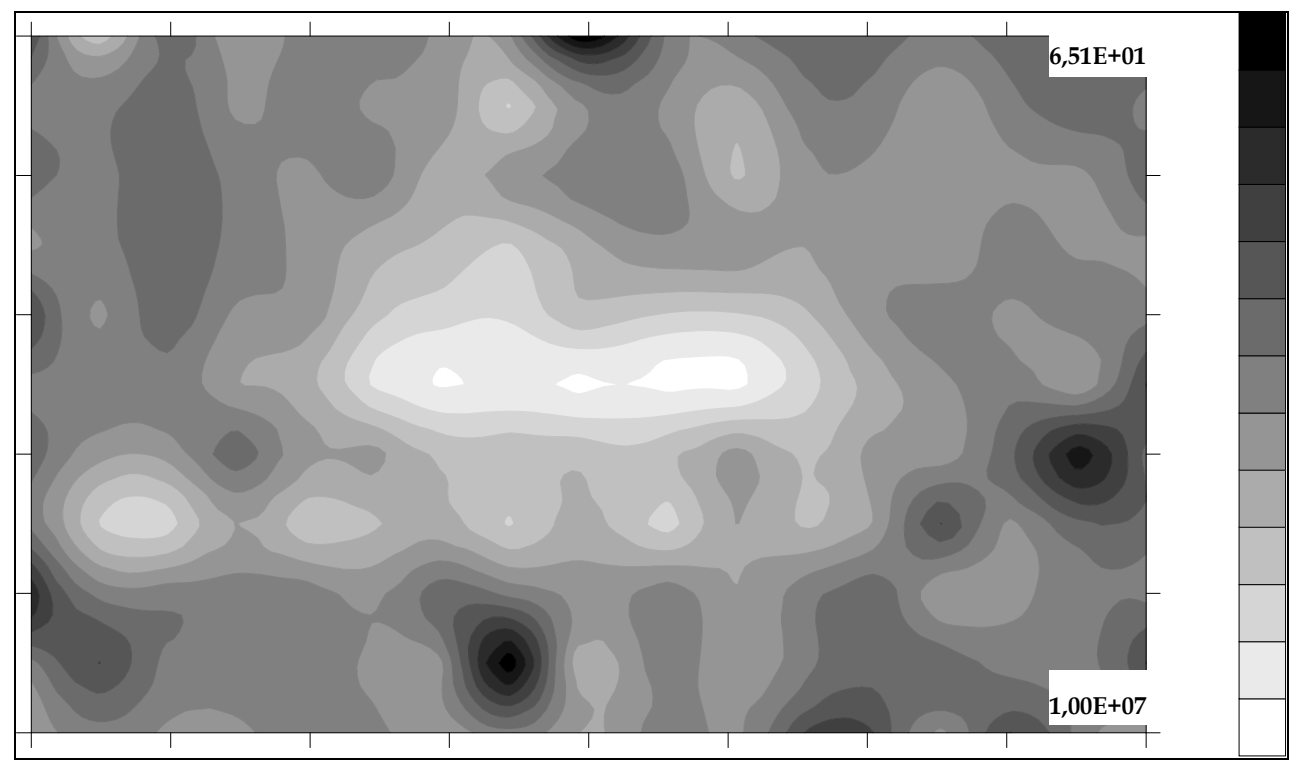

Fig. 14. Signal power $[\mathrm{mV}]$ : distribution map.

Figures 15 and 16 show respectively the results of the performed Travel Time and Spectral Attenuation Tomography measurements on the selected horizontal section of the full-scale real masonry (Concu et al., 2010). Showed results of Spectral Attenuation AT have been achieved by using the sweep signal as source.

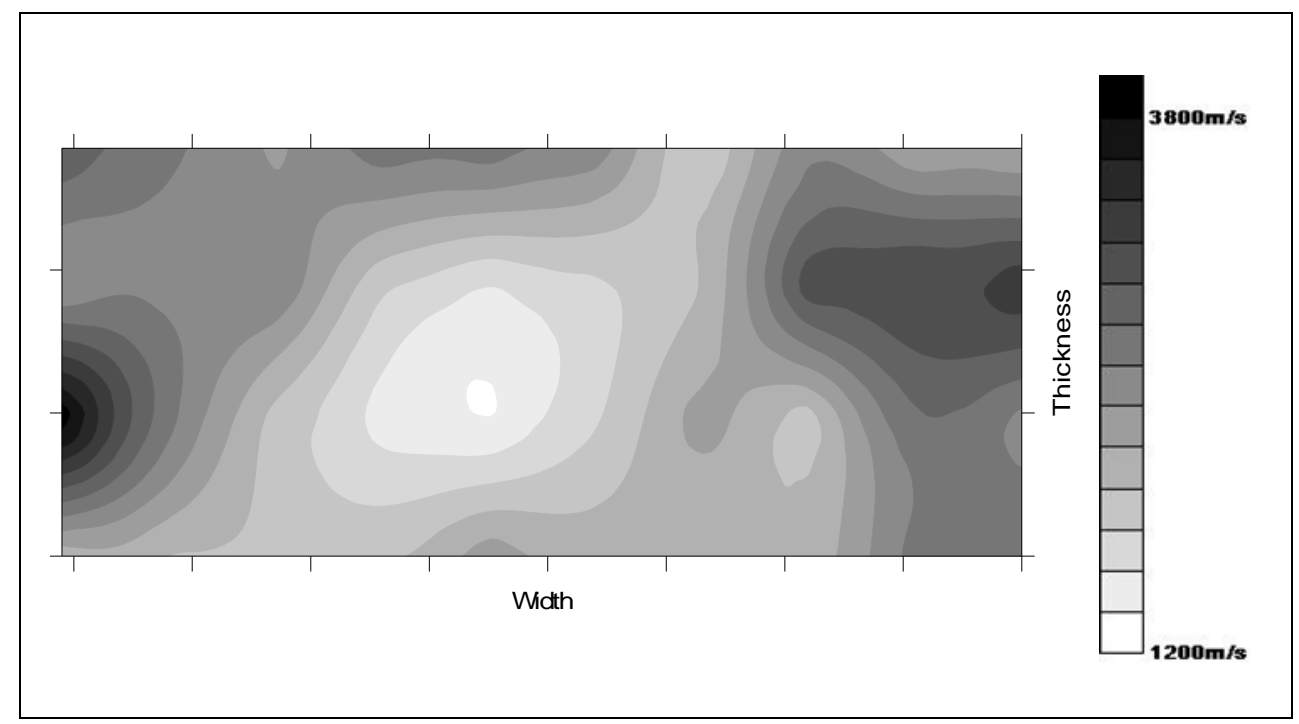

Fig. 15. Travel Time Tomography measurements 


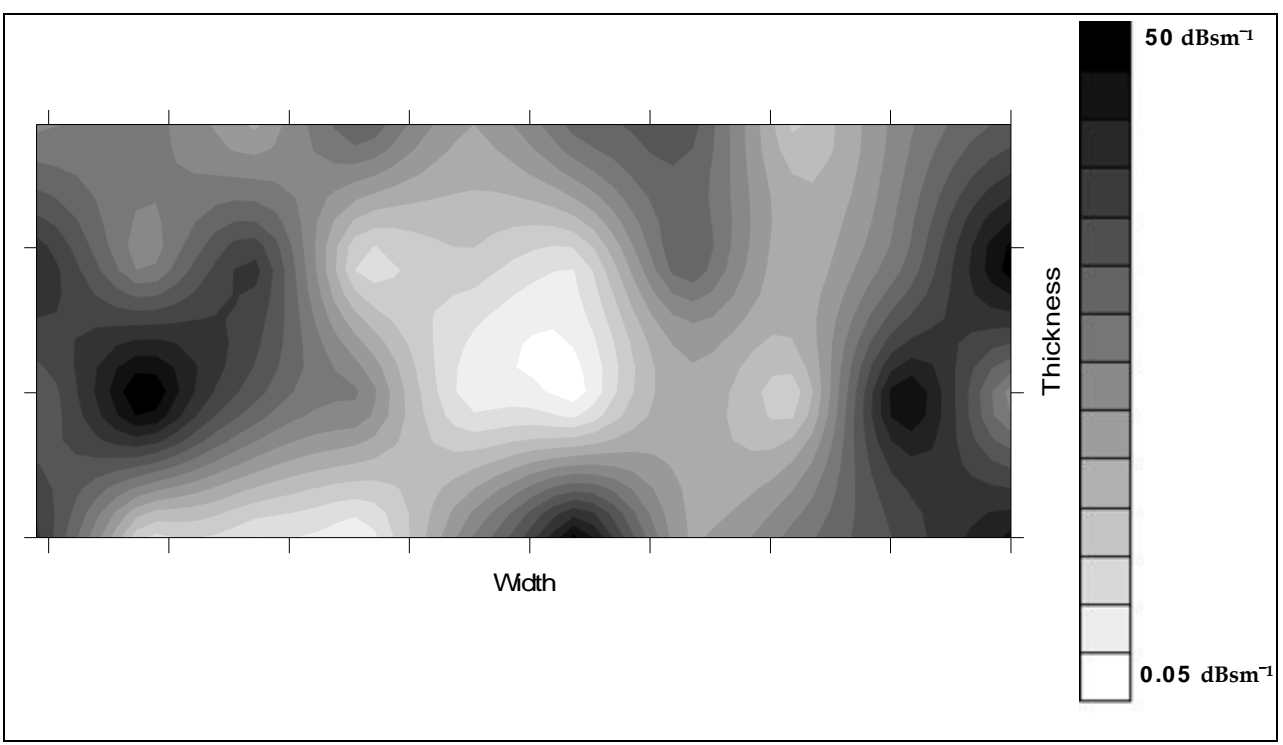

Fig. 16. Spectral Attenuation Tomography measurements

It can be noticed that both maps identify a central region corresponding to the position of the real cavity. The velocity map presents a poor quality in terms of the position and also the extension of the cavity, both parameters being significantly improved by using the attenuation map. It can be also seen that the attenuation map presents a high degree of scattering, confirming the hypothesis that this parameter is very sensible to all discontinuities of a section such as joints, small irregularities, various interfaces. It is worth noting that a tighter grid of measurements would enable a better definition of the cavity's shape, implying longer measuring and computational times.

Results of DTT and AT thus emphasize the main role played by frequency dependent features, such as signal power and spectral attenuation coefficient, when dealing with materials and structures with a quite important degree of non homogeneity. It is worth stressing that the maps derived from DTT do not allow the anomaly to be collocated at its real and correct distance from the wall surface, while the AT maps give a faithful picture of the selected section.

\section{Comments on measurements problems}

Acoustic measurements are still affected by a quite large amount of uncertainness in terms of stability and reproducibility. This is primarily due to the large number of factors that somehow exert influence on measurements:

- materials (chemical-physical-mechanical conditions);

- signals;

- $\quad$ acoustic parameters;

- instrumental sets;

- data processing methods; 
- environmental conditions;

- human factor.

Another big problem concerning acoustic measurements is the gap that exists between the theoretical and interpretative bases and the codification of operative modalities, which is to say the almost total lack of standardization procedures. Actually, only standards concerning the determination of ultrasonic pulse velocity in concrete and natural stones are available (EN 12504-4, 2004; EN 14579, 2004). These standards take into account the problem of measurements stability, repeatability and reproducibility, and address the main factors influencing pulse velocity measurements. It is stated that to obtain some measure of the acoustic velocity that is reproducible and which is essentially a function of the properties of the material (concrete and stone) submitted to testing, it is necessary to take into consideration the different factors that exert an influence on the velocity. This is also essential to establish the correlations existing with the different physical characteristics of the material. The factors that should be carefully considered are the following:

a. moisture content and temperature of the concrete

b. water content of the stone

c. path length

d. shape and size of the specimen

e. cracks, fissures and voids.

- Moisture and temperature of the concrete and water content of the stone

Moisture content has both chemical and physical effects on ultrasonic pulse velocity. These effects are important especially for establishing correlations with concrete strength. As an example, acoustic velocity can vary significantly between a properly cured standard specimen and a structural element made of the same concrete, because of the influence of curing conditions on cement hydration and the presence of free water in the voids. In the same way, water content has some effects on pulse velocity propagation in stones. Stone humidity, that is to say the presence of water inside the pores, can cause a variation of ultrasonic velocity value up to $50 \%$ with respect to dry specimens or structural components. Concrete temperature effects on ultrasonic pulse velocity should be taken into account only outside the range $10^{\circ} \mathrm{C}-30^{\circ} \mathrm{C}$. Within this range, no significant change in pulse velocity has been experimentally found if corresponding changes in strength or elastic properties do not occur.

- Path length, specimen shape and size

The path length over which the pulse velocity is measured should be long enough not to be significantly influenced by the heterogeneous nature of the concrete or the stone. For concrete specimen the standard recommends minimum values of the path length depending on aggregates nominal maximum size. The velocity is not generally affected by the variations in the path length, even though the electronic timing devices are likely to give some indications that the velocity can slightly decrease if the length of the path increases. This is because the higher frequency components of the pulse are attenuated more than that lower frequency components and the shape of the onset of the pulse becomes more rounded with increased distance travelled. Thus, the apparent reduction of pulse velocity arises from the difficulty of defining exactly the onset of the pulse and this depends on the particular method used for its definition. The apparent reduction in velocity is normally slight and is within the accuracy of measurements commercial apparatus. Nevertheless, particular care shall be taken when measurements are carried out on important path lengths. The velocity of short vibratory impulses is independent 
of the size and shape of specimen in which they travel, unless its least lateral dimension is less than a minimum value. Below this value, the pulse velocity can be reduced appreciably. The amount of this reduction depends primarily on the ratio ultrasonic wavelength to smallest lateral dimension of the specimen, but is insignificant if the ratio is less than unity. Standards give the relationships existing between pulse velocity, transducers frequency and the smallest admissible lateral dimension of the test specimen. If the minimum lateral dimension is less than the wavelength or if the indirect transmission arrangement is used, propagation mode changes and, therefore, measured velocity will be different. This is particularly important in cases where elements of significantly different sizes are being compared.

- Cracks, fissures and voids

When a wave passing through a specific item encounters any discontinuity, the wave power is most likely attenuated because of scattering phenomena, so that any crack, fissure or void inside the item might obstacle wave propagation. The higher the projection of defects length with respect to transducers size and wavelength, the greater the influence on wave propagation. In this case, the first impulse picked up by the receiving transducer will undergo a diffraction at the edge of the anomaly and this gives a longer path time compared to a transmission taking place in items with no fissures or voids. If part of the signal is already able to reach the receiver - e.g. in cracked elements where the broken sides are kept firmly in contact by compression then the propagation time may not significantly change with respect to paths with no defects throughout. Transit time is not a decisive parameter also when the crack is filled with a liquid able to transmit wave power. In all these cases, examination of signal attenuation may also provide helpful information. Therefore, it would be reasonable to approach the ultrasonic analysis not only in terms of propagation times, but also in terms of other wave's features changes. The higher the intrinsic non-homogeneity level of structures, e.g. masonries, the greater the advisability of this integrated approach.

The different applications of sonic and ultrasonic investigations have brought to the fore two fundamental issues in the performance of such tests and their repeatability: the influence of pressure exerted on the transducers employed and the effect on acquired data of the application of acoustic coupling agents inserted between the material under examination and the transducers.

Experiments performed on granite specimens in different operative conditions, that is, in presence or absence of the acoustic coupling agent and of pressure on the transducers, allowed us to evaluate the impact of these factors on the characterization of the material and the repeatability of measurements (Concu, 2002). It emerged that the best operating conditions, which ensure excellent measurement repeatability and reliability, are those that call for both the use of the coupling agent placed between the surface of the material and the transducers used for measuring and the application of a constant pressure on the transducers throughout the entire test period. The uncertainty introduced by the variability in pressure and the conditions of the coupling agent (thickness, temperature, viscosity) is in fact converted into a variation of the energy associated with the transmitted signal; this impacts to a greater degree on the wave shape and the signal spectrum rather than on its propagation velocity. From the experiments performed another interesting datum emerged, that is, the influence on the measurement of time between application of the acoustic coupling and data acquisition (Concu \& Fais, 2003). Analysis of the values of maximum wave amplitude, amplitude of the maximum spectral peak and velocity relating to 
ultrasonic signals acquired repeatedly over time, starting from the instant of application of the coupling agent, led to the observation of a behavior of the aforementioned characteristic parameters of the material under examination: as can be seen in Figures 17-19 the three parameters increase progressively for approximately the first sixty minutes from application of the acoustic coupling agent and then become constant. Such behavior is to be taken into account in defining for each tested material the optimal instant for data acquisition so as to reduce the influence of the coupling agent on the test and its repeatability.

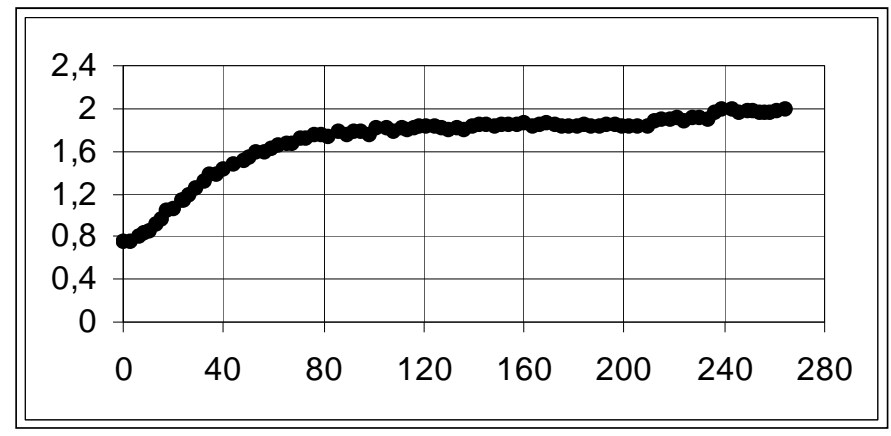

Fig. 17. Maximum wave amplitude [V] vs time [minutes]

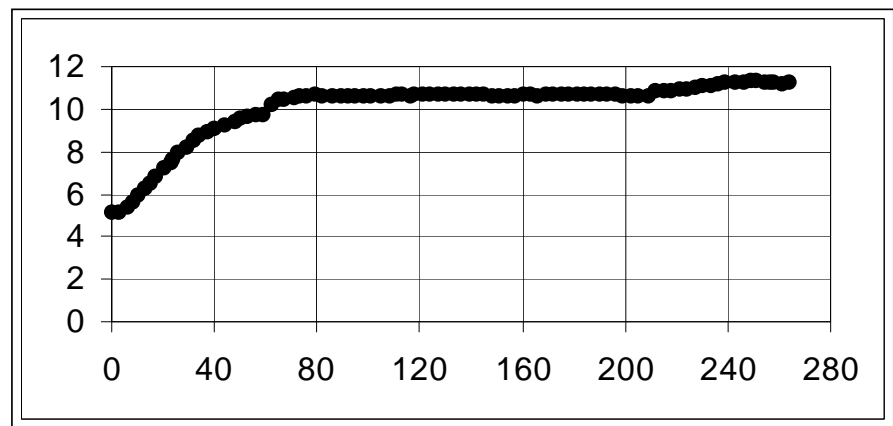

Fig. 18. Amplitude of the maximum spectral peak vs time [minutes]

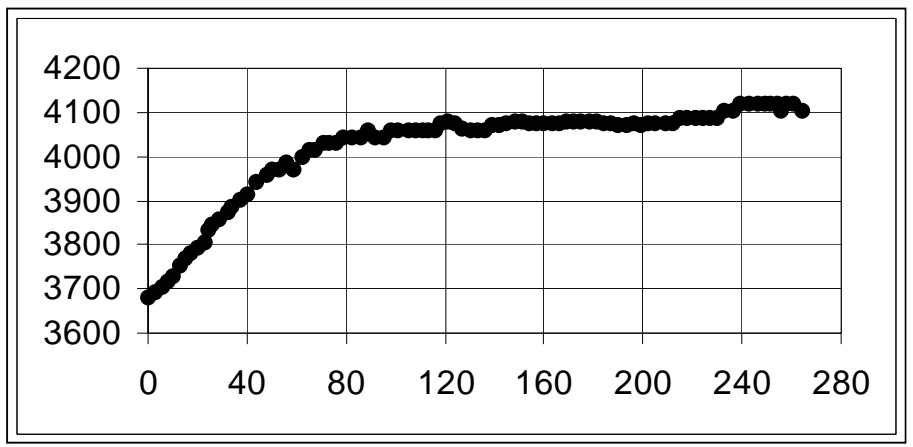

Fig. 19. Propagation velocity $[\mathrm{m} / \mathrm{s}]$ vs time [minutes] 


\section{Conclusions}

The chapter presented an overview on non invasive acoustic measurements applied for faults detecting in building materials and structures. The state of art has been described, focusing on two main operative procedures: the Direct Transmission Technique and the Acoustic Tomography. Special emphasis has been dedicate to both Travel Time and Spectral Attenuation Tomography, by deepening the aspects of numerical modeling, resolution algorithms, choice of source signal, measuring systems setup.

A case study, reporting the results of an experimental program started with the aim of deepening the reliability of acoustic methods in buildings faults detecting and materials characterization, has been described. Both DTT and AT approaches have been applied to a full scale masonry model with known anomalies inside. Results showed that both DTT and AT can successfully apply to stone masonry characterization. Moreover, results confirmed the suitability of the Spectral Attenuation Tomography - developed according to the model proposed by Quan and Harris, 1997, for seismic surveying - in buildings materials and structures faults detection.

Finally, an outline of the most common problems affecting the acoustic non destructive testing has been illustrated, addressing the main factors influencing the acoustic measurements in terms of stability, repeatability and reproducibility. It has been pointed out that particular attention should be given to chemical-physical-mechanical conditions of the material and to testing modalities such as the pressure exerted on the transducers employed and the effect on acquired data of acoustic coupling agents.

Further researches should deepen various facets, including:

- the potentiality of a novel approach involving the integrate analysis of different features, associated with acoustic waves propagating through the material, acquired both in time and frequency domain;

- the implementation of different inversion algorithms, chosen among the most robust and commonly used, for tomographic measurements, with the aim of highlighting the most suitable one for the specific algebraic problem solution;

- the development of proper measurements setup and automated measuring procedure which allowed problems of measurements stability, repeatability and reproducibility to be minimized.

\section{References}

Belanger, P.\& Cawley, P. (2009). Feasibility of low frequency straight-ray guided wave tomography. NDTEE International, Vol.42, No.2, March 2009, pp. 113-119

Berryman, J.G. (1991). Non linear inversion and tomography. Lecture notes, Earth Resource Laboratory, MIT

Best, A.I., McCann, C. \& Sothcott, J. (1994). The relationships between the velocities, attenuations, and petrophysical properties of reservoir sedimentary rocks. Geophysical Prospection, Vol.42, No.2, February 1994, pp. 151-178 
BS 1881: Part 203, Recommendations for measurement of the velocity of ultrasonic pulses in concrete, London, 1986

Cannas, B., Cau, F., Concu G. \&Usai M. (2008). Features extraction techniques for sonic and ultrasonic NDT on building materials. Proceedings of $1^{\text {st }}$ International Symposium on Life-Cycle Civil Engineering, ISBN 978-0-415-46857-2, Varenna, Lake Como, Italy, June, 2008

Carino, N.J. (2001). The Impact-Echo Method: An Overview. Proceedings of the 2001 Structures Congress \& Exposition, Washington, D.C., May 21-23, 2001

Concu, G. (2002). Il problema del controllo delle prestazioni degli elementi lapidei impiegati nelle costruzioni, $\mathrm{PhD}$. Thesis, University of Cagliari, Italy

Concu G., De Nicolo B. \& Mistretta F. (2003a). Comparative evaluation of two nondestructive acoustic techniques applied in limestone masonry diagnosis, Proceedings of 33 International Conference and Exhibition Defectoscopy, ISBN 80-2142499-0, Ostrava, Czech Republic, November, 2003

Concu G., De Nicolo B., Mistretta F.\& Valdés M. (2003b). NDT ultrasonic method for ancient stone masonry diagnosis in Cagliari (Italy), Proceedings of Structural Faults and Repair - 2003, ISBN 0-947644-52-0, London, UK, July, 2003

Concu, G. \& Fais S. (2003). In time analysis of a viscous coupling agent effect in ultrasonic measurements. Proceedings of 3rd International Conference on Non-Destructive Testing of the Hellenic Society for NDT - NDT in Antiquity and Nowadays - Skills-ApplicationsInnovations, Chania, Crete, Greece, October, 2003

Concu, G., De Nicolo, B., Piga, C. \& Popescu, V. (2010). Measurement system for nondestructive testing using ultrasonic tomography spectral attenuation. Proceedings of $12^{\text {th }}$ International Conference on Power Electronics and Electrical Engineering, ISBN 9781-4244-7020-4, Brasov, Romania, May, 2010

Dines, K.A. \& Lytle, R.J. (1979). Computerized geophysical tomography. Proceedings of IEEE 67(7), pp. 1065-1073

Enoki, M. \& Kishi, T. (1991). Acoustic Emission. Current Practice and Future Directions, ASTM, Philadelphia

EN 12504-4 (2004). Testing concrete- Part 4. Determination of ultrasonic pulse velocity

EN 14579. (2004). Natural stone test methods. Determination of sound speed propagation

Gilbert, P. (1972). Iterative methods for the reconstruction of three-dimensional objects from projections. Journal of Theoretical Biology, Vol.36, No.1, July 1972, pp. 105117

Gordon, R., Bender, R. \& Herman, G.T. (1970). Algebraic Reconstruction Technique (ART) for three-dimensional electron microscopy and x-ray photography, Journal of Theoretical Biology, Vol.29, No.3, December 1970, pp.471-81

Gordon, R. (1974), A tutorial on ART. Proceedings of IEEE Trans. Nuclear Science, NS-21, pp. 78-93

Herman, G.T. (1980). Image Reconstruction from Projection: the Fundamentals of Computerized Tomography, Academic, New York

Hudson, J.A. (1981). Wave speeds and attenuation of elastic waves in material containing cracks. Geophysical Journal International, Vol.64, no.1, January 1981, pp. 13350 
Ilyas, A. (2010). Estimation of Q factor from reflection seismic data, Report GPM 6/09, Department of Exploration Geophysics, Curtin University of Technology, p. 65

Ivansson, S. (1986). Seismic Borehole Tomography - Theory and Computational Methods. Proceedings of IEEE 74, pp. 328-338

Jansen, D.P., Hutchins, D.A., Ungar, P.J. \& Young R.P. (1991). Acoustic tomography in solids using a bent ray SIRT algorithm. Nondestructive Testing and Evaluation, Vol.6, No.3, pp. 131-148

Johnston, D.H. (1981). Attenuation: A state-of-art summary. In Toksöz and Johnston, Eds., Geophysics reprint series, Vol.2, Soc. of Expl. Geophys

Kaiser, J. (1950). An Investigation into the Occurrence of Noises in Tensile Tests or a Study of Acoustic Phenomena in Tensile Tests. Ph.D. Thesis, Tech. Hosch. Munchen, Munich, Germany

Kepler, W.F., Leonard Bond, J. \& Frangopol, D.M. (2000). Improved assessment of mass concrete dams using acoustic travel time tomography. Part II: application. Construction and Building Materials, Vol.14, No.3, April 2000, pp. 147-156

Knopoff, L (1964). The Convection Current Hypothesis. Reviews of Geophysics, Vol.2, No.1, pp. 89-122

Krautkramer, J.\& Krautkramer, H. (1990). Ultrasonic testing of materials, Springer Verlag, New York

Lakshiminarayanan, V.A. \& Lent A. (1979). Methods of least squares and SIRT in reconstruction. Journal of Theoretical Biology, Vol.76, No.3, February 1979, pp. 267295

Jansen Leonard Bond, J., Kepler, W.F. \& Frangopol, D.M. (2000). Improved assessment of mass concrete dams using acoustic travel time tomography. Part I: theory. Construction and Building Material, Vol.14, No.3, April 2000, pp. 133-146

Lo, T.W., Inderwiesen, P. (1994). Fundamentals of Seismic Tomography. Soc. of Expl. Geoph., Tulsa

Meglis, I.L., Chow, T., Martin, C.D. \& Young, R.P. (2005). Assessing in situ microcrack damage using ultrasonic velocity tomography. International Journal of Rock Mechanics \& Mining Sciences, Vol.42, No.1, January 2005, pp. 25-34

NDT Education Resource Center. Larson, B. Editor. (2001-2011). The Collaboration for NDT Education. Iowa State University. Available at: $<$ www.ndt-ed.org>

Ohtsu, M. \& Ono K. (1986). The generalized theory and source representations of acoustic emission. J. of Acoustic Emission, Vol. 5, No. 4, October-December 1986, pp. 124133

Priestley, M.B. (1981). Spectral Analysis and Time Series. vol. I, Academic Press, New York

Quan, Y. \& Harris, J.M. (1997). Seismic attenuation tomography using the frequency shift method. Geophysics, Vol.62, No.3, May-June 1997, pp. 895-905

Rhazi, J. (2006). Evaluation of concrete structures by the Acoustic Tomography Technique. Structural Health Monitoring, Vol.5, no.4, December 2006, pp. 333-342

Sansalone, M. \& Streett, W.B. (1997). Impact-Echo: Nondestructive Evaluation of Concrete and Masonry. Ithaca, N.Y., Bullbrier Press

Sheriff, R.E. \& Geldart L.P. (1995). Exploration Seismology. Cambridge Univ. Press, London 
UNI EN 1926 (2000). Natural stone test methods. Determination of compressive strength UNI EN 14580 (2005). Natural stone test methods. Determination of static elastic modulus 


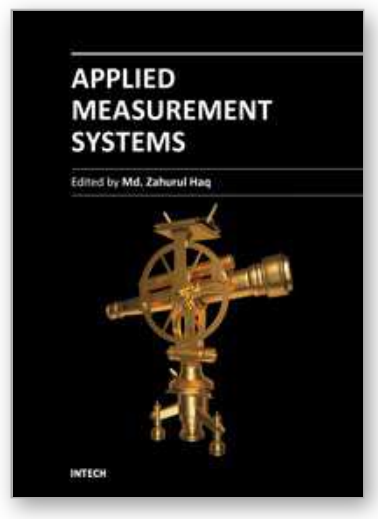

\author{
Applied Measurement Systems \\ Edited by Prof. Zahurul Haq
}

ISBN 978-953-51-0103-1

Hard cover, 390 pages

Publisher InTech

Published online 24, February, 2012

Published in print edition February, 2012

Measurement is a multidisciplinary experimental science. Measurement systems synergistically blend science, engineering and statistical methods to provide fundamental data for research, design and development, control of processes and operations, and facilitate safe and economic performance of systems. In recent years, measuring techniques have expanded rapidly and gained maturity, through extensive research activities and hardware advancements. With individual chapters authored by eminent professionals in their respective topics, Applied Measurement Systems attempts to provide a comprehensive presentation and in-depth guidance on some of the key applied and advanced topics in measurements for scientists, engineers and educators.

\title{
How to reference
}

In order to correctly reference this scholarly work, feel free to copy and paste the following:

Barbara De Nicolo, Carlo Piga, Vlad Popescu and Giovanna Concu (2012). Non Invasive Acoustic Measurements for Faults Detecting in Building Materials and Structures, Applied Measurement Systems, Prof. Zahurul Haq (Ed.), ISBN: 978-953-51-0103-1, InTech, Available from: http://www.intechopen.com/books/applied-measurement-systems/non-invasive-acoustic-measurements-forfaults-detecting-in-building-materials-and-structures

\section{INTECH}

open science | open minds

\author{
InTech Europe \\ University Campus STeP Ri \\ Slavka Krautzeka 83/A \\ 51000 Rijeka, Croatia \\ Phone: +385 (51) 770447 \\ Fax: +385 (51) 686166 \\ www.intechopen.com
}

\author{
InTech China \\ Unit 405, Office Block, Hotel Equatorial Shanghai \\ No.65, Yan An Road (West), Shanghai, 200040, China \\ 中国上海市延安西路65号上海国际贵都大饭店办公楼 405 单元 \\ Phone: +86-21-62489820 \\ Fax: +86-21-62489821
}


(C) 2012 The Author(s). Licensee IntechOpen. This is an open access article distributed under the terms of the Creative Commons Attribution 3.0 License, which permits unrestricted use, distribution, and reproduction in any medium, provided the original work is properly cited. 